\title{
The Regulation of Developmental Diapause is Coordinated with Molting and Olfaction
}

Heather R. Carstensen ${ }^{1}$, Reinard M. Villalon ${ }^{1}$, Johnny Vertiz ${ }^{1}$, Navonil Banerjee ${ }^{2}$, Elissa A. Hallem², and Ray L. Hong ${ }^{1, *}$

${ }^{1}$ California State University, Northridge

Department of Biology

18111 Nordhoff Street

Northridge CA, 91330-8303, USA

${ }^{2}$ University of California, Los Angeles

Dept. of Microbiology, Immunology \& Molecular Genetics and Molecular Biology Institute 615 Charles E. Young Drive S.

Los Angeles, CA 90095, USA

*Corresponding author: ray.hong@csun.edu

Keywords: dauer larva, evolution of development, nematodes, steroid hormones, cuticle exsheathment, olfaction

Abbreviations: dauer larva (DL); (Z)-7-tetradecen-2-one (ZTDO);

Author contributions: HRC, RMV, NB, and RLH designed and performed the experiments; JV performed the experiments; HRC, RMV, JV, NB, and RLH analyzed data; HRC, RV, and RLH wrote the manuscript.

The authors declare no conflict of interest. 


\section{Abstract}

2 Developmental and behavioral plasticity allow animals to prioritize alternative

3 genetic programs during fluctuating environments. Behavioral remodeling may be

4 acute in animals that interact with host organisms, since reproductive adults and

5 the developmentally arrested larvae often have different ethological needs for

6 chemical stimuli. To understand the genes that coordinate development and

7 behavior, we used the nematode model Pristionchus pacificus to characterize

8 mutants that inappropriately enter developmental diapause to become dauer

9 larvae (Daf-c). We found several key olfactory differences between $P$. pacificus

10 and C. elegans Daf-c dauers. In addition, the two $P$. pacificus Daf-c alleles disrupt

11 steroid synthesis required for proper regulation of the conserved canonical steroid

12 hormone receptor DAF-12, whose dauer-constitutive and cuticle exsheathment

13 phenotypes can be rescued by the feeding of $\Delta 7$-dafachronic acid. One allele,

14 csu60, has a deletion in the sole HydroxySteroid Dehydrogenase (HSD) in $P$.

15 pacificus. Both hsd-2(csu60) adults and dauers show enhanced attraction to a

16 beetle pheromone, possibly due to the heterochronic activation of dauer-specific

17 neuronal development in the adults. Surprisingly, this enhanced odor attraction

18 acts independently of daf-12, revealing unexpected targets of steroid hormones

19 regulating ecdysis and olfaction in $P$. pacificus. 


\section{Author Summary}

21 The remarkable evolutionary success of nematodes can be attributed to their

22 dispersal ability as stress-resistant dauer larvae and the equivalent parasitic

23 infective larvae. The decision to enter dauer development is regulated by a

24 conserved steroid hormone receptor that receives multiple external and internal

25 cues, though the extent in which these cues also coordinate other physiological

26 and behavioral processes is not well understood in divergent species. We used

27 the insect-associated nematode Pristionchus pacificus to genetically dissect two

28 mutants that form dauers inappropriately, and found that one mutation in a

29 steroidogenic enzyme, Ppa-hsd-2, is predicted to abolish the biosynthesis of the

30 hormones needed to negatively regulate dauer entry when food is available.

31 Unexpectedly, Ppa-HSD-2 is also required to properly complete the dauer larval

32 molt, known as exsheathment, as well as to confer differences in adult versus

33 dauer larvae chemotaxis behavior towards a host pheromone. Given that dauers

34 are the host-seeking stage of parasitic nematodes, hormonal disruption represents

35 a tenable target for biological control. 


\section{Introduction}

37 Organisms integrate multiple sensory stimuli continuously from their environment

38 to make key developmental decisions and engage in context-dependent behavior.

39 When environmental conditions are unfavorable, a portion of the population can

40 stop feeding and suspend development. In nematodes, of the several larval stages

41 that can undergo developmental arrest [1,2], the dauer larvae (DL) in

42 Caenorhabditis elegans, as well as the equivalent infective juvenile (IJ) or infective

43 third-larval (iL3) stages in parasitic nematodes, undergo spectacular physiological,

44 morphological and neuroanatomical changes [3-5]. At the gene expression level,

45 a number of the dauer entry changes are reversible while some become

46 permanent life history traits that mark dauer passage [6,7]. Entry into the dauer

47 stage is primarily determined by environmental factors, particularly the depletion

48 of food, elevated temperature, and high levels of ascaroside dauer pheromones in

49 response to overcrowding (Cassada and Russel, 1975; Golden and Riddle 1982

50 and 1984a; Jeong et al., 2005; Butcher et al., 2007). The DL is thus a striking

51 example of developmental plasticity that is becoming more amenable to genetic

52 analysis in diverse species of nematodes as a result of comparative studies in non-

53 Caenorhabditis species [8-10].

$54 \quad$ The commitment to dauer entry requires external as well as internal factors.

55 Multiple parallel-acting signaling factors in the dauer regulation pathway have been

56 characterized in C. elegans, such as the recognition of pheromones by G-protein-

57 coupled receptors in the chemosensory amphid neurons and signal transduction

58 to the DAF-11/guanylyl cyclase pathway [11]. Favorable conditions also lead to 
59 signaling in the DAF-7/TGF-ß and DAF-2/insulin-like pathways [11-14]. This

60 signaling facilitates the production of $\Delta 4$ - and $\Delta 7$-dafachronic acid from the

61 cholesterol precursor, which act as ligands that bind to the highly conserved

62 nuclear hormone receptor DAF-12 and prevent DAF-12 from repressing genes

63 necessary for development to reproductive adulthood [11,15-17]. Less favorable

64 conditions produce pheromones that reduce signaling in these pathways,

65 subsequently shifting DAF-12 from its inactive ligand-bound form to its active,

66 ligand-free form that specifies dauer development. Despite being a non-feeding

67 and stress-resistant stage, the DL are actively motile and receptive to external

68 chemosensory and mechanical cues. For both parasitic and free-living nematodes,

69 DL share the ability to survive harsh conditions but remain receptive to

70 semiochemicals from their environment.

71 The commitment to dauer also prompts the rewiring of the sensory nervous

72 system in C. elegans $[6,18,19]$, but the molecular basis of this process remains

73 poorly understood [20]. Comparisons of chemosensory behaviors in reproductive

74 free-living adults versus developmentally arrested DL or iL3s have revealed that

75 responses to some chemosensory cues change dramatically across life stages.

76 For example, C. elegans well-fed adults are repelled by carbon dioxide $\left(\mathrm{CO}_{2}\right)$,

77 while $C$. elegans DL are attracted to $\mathrm{CO}_{2}[21,22]$. However, most genetic studies

78 of chemosensory behavior have been conducted on young adults in free-living

79 nematodes [23-25], while chemotaxis behaviors in parasitic nematodes were

80 profiled primarily with IJs or iL3s [26-31]. 
81 To better understand the genetic network that coordinates dauer entry with

82 development in species that evolved in fluctuating environments with hosts, the

83 entomophilic nematode Pristionchus pacificus has been cultivated as a genetically

84 tractable comparison to $C$. elegans. In the wild, $P$. pacificus has been found as DL

85 on various species of beetles, which upon the hosts' death, exit the dauer state

86 and resume reproductive development to feed on the bacteria [32-34]. Thus,

87 Pristionchus DL are considered to be the host-seeking stage, with adaptations that

88 include an oily coat that promotes aggregation, survival up to a year (perhaps due

89 to the host life cycle), and mouth form plasticity, in which dauer passage increases

90 the proportion of the bacteriovorous stenostomatous form with faster pharyngeal

91 pumping [35-38]. Furthermore, adult Pristionchus species have distinctive

92 chemosensory preferences for high molecular weight insect pheromones and plant

93 volatiles that differ significantly from Caenorhabditis species, and also differ across

94 Pristionchus species [39]. However, due to the low number of DL that can be

95 induced by starvation in the reference strain, the chemosensory profiles of $P$.

96 pacificus DL have not yet been determined.

97 In P. pacificus, several dauer formation defective (Daf-d) mutants that fail

98 to form DL under inducing conditions have been isolated by unbiased forward

99 genetics as well as reverse genetics. These studies have identified genes

100 encoding for the nuclear hormone receptor DAF-12 and the forkhead transcription

101 factor DAF-16 as important regulators of dauer formation [8,9]. However, while the

102 DAF-12 and DAF-16 functions are conserved between C. elegans and P. pacificus,

103 mutations in other Daf-d orthologs suggest divergence in the factors that act 
104 upstream of these proteins. In C. elegans, high population density induces the 105 secretion of dauer pheromones consisting of a modular library of ascarosides [40-

106 43], and mutations in the ascaroside biosynthesis enzyme, DAF-22, result in the

107 Daf-d phenotype [44]. Interestingly, the daf-22 gene underwent a recent 108 duplication in $P$. pacificus that resulted in subfunctionalization in ascaroside 109 production, yet neither mutations in the Ppa-daf-22.1/daf-22.2 paralogs alone or in 110 combination resulted in defects in dauer formation [45]. These studies indicate a 111 possible significant divergence in $P$. pacificus and $C$. elegans dauer regulation 112 upstream of the conserved DAF-12 and DAF-16 modules.

113 In contrast to the Daf-d mutants, no Dauer formation constitutive (Daf-c)

114 mutants have been characterized extensively in $P$. pacificus, despite numerous

115 Daf-c orthologs in the genome. Putative homologs of the $C$. elegans DAF116 11/guanylyl cyclase, DAF-7/TGF-ß ligand, and DAF-2/insulin receptor have been 117 identified in the $P$. pacificus genome (www.wormbase.org), but it remains unknown

118 if any of these homologs are involved in dauer regulation. At the same time,

119 targeted mutations in $P$. pacificus orthologs of other $C$. elegans Daf-c mutants did 120 not result in dauer formation phenotypes. Specifically, mutations in the P. pacificus

121 heat shock protein Ppa-DAF-21/HSP90 and Ppa-DAF-19/RFX transcription factor 122 affected ciliogenesis but did not result in a Daf phenotype [46,47]. Copy number 123 variations in the $P$. pacificus dauerless locus, a fast-evolving gene with homologs 124 only in the Pristionchus genus, control responsiveness to dauer pheromones but 125 do not cause a Daf-c phenotype [48]. Finally, because cholesterol is a precursor 126 for the synthesis of steroid hormones needed for both proper ecdysis and dauer 
127 formation $[49,50]$, mutations in cholesterol metabolism and steroid signaling would

128 be expected among yet-undescribed Daf-c mutants.

129 To identify the key molecular factors controlling dauer-specific phenotypes,

130 we examined the development and behavior of $P$. pacificus mutants that form DL

131 constitutively. In a previous study, a forward genetic screen in $P$. pacificus yielded

132 four dauer constitutive Daf-c alleles, $d f c$ (dauer-formation constitutive), which can

133 be rescued by exogenous $\Delta 7$-dafachronic acid [8,51]. One of the strongest alleles,

134 Ppa-dfc-1(tu391), has been positionally mapped, but the genetic lesion remains

135 unknown. We therefore isolated a new Daf-c allele, csu60, and following positional

136 mapping and whole genome sequencing, found that csu60 is a null allele of the

137 sole hydroxysteroid dehydrogenase in $P$. pacificus, an enzyme responsible for the

138 production of dafachronic acids. For both Daf-c alleles, the DL and adults have

139 olfactory behavior resembling wild-type starvation-induced DL, suggesting that the

140 genes which control dauer entry also normally repress host-seeking olfactory

141 behavior in adults. 


\section{Results}

\section{P. pacificus Daf-c mutants have an exsheathment phenotype}

$144 P$. pacificus dauer larvae (DL) can be induced by starvation, crowding, or high

145 temperature $\left(\geq 25^{\circ} \mathrm{C}\right)$. We characterized two incompletely penetrant, recessive,

146 non-allelic dauer formation-constitutive (Daf-c) mutants at $20^{\circ} \mathrm{C}$, Ppa-dfc-1(tu391)

147 and csu60. Like several species of parasitic nematodes, most wild-type $P$. 148 pacificus DL are ensheathed (63\%), where the sheath is the apolysed but

149 unecdysed cuticle of the J2 larvae [52]. Strikingly, while the ensheathed wild-type

150 DL are motile, both Daf-c alleles exhibited an unusual ensheathment-related defect

151 in which live worms are trapped and immobilized inside an old cuticle (Fig. 1A-C)

152 [53]. Aside from the loosened cuticle, most "incarcerated" larvae in tu391 and

153 csu60 are indistinguishable from DL produced by the wild-type reference strain

154 PS312 under starvation condition: they are characterized by radial shrinkage of 155 the body, a darkly hued gut, and a buccal cap covering the mouth. In

156 entomopathogenic nematodes from the genera Steinernema and Heterorhabditis,

157 ensheathment appears to confer increased ability to resist desiccation, while 158 exsheathment leads to increased motility and is the first step of the host infection 159 process [53,54]. Our results reveal that in $P$. pacificus, exsheathment is genetically 160 coupled with dauer entry.

161 While most of the csu60 incarcerated larvae resemble DL, some animals 162 show less radial constriction than wild-type dauers. To confirm that all incarcerated 163 worms are exclusively in the dauer stage, we measured their pharyngeal pumping 164 frequency. We found that the incarcerated tu391 and csu60 larvae display 
165 pumping rates significantly lower than the $\mathrm{J} 3$ and $\mathrm{J} 4$ stages of wild-type and mutant

166 animals, but indistinguishable from their respective non-incarcerated (active) DL

167 as well as wild-type $P$. pacificus and C. elegans DL (Fig. 1D). We isolated tu391

168 and csu60 J3 and J4 larvae onto fresh OP50 to determine if they develop into

169 incarcerated larvae but did not observe incarcerated worms. Furthermore, we

170 transferred mutant tu391 J2 larvae onto fresh E. coli OP50 and found that J2 larvae

171 formed J3 larvae, active DL, or incarcerated DL, suggesting that the molting defect

172 in tu391 and csu60 is exclusive to the J2-DL transition.

173 To determine if the incarcerated DL is a permanent or transient phenotype,

174 we picked incarcerated tu391 and csu60 DL onto fresh OP50 plates. Because

175 transferring the incarcerated DL may trigger the release the trapped $\mathrm{DL}$, we also

176 isolated the incarcerated tu391 DL by removing all other worms from the plate

177 rather than picking the incarcerated tu391 DL. Surprisingly, all 'disturbed' and 178 'undisturbed' incarcerated tu391 and csu60 DL became free and active within 24

179 hours, regardless of whether they were maintained at $20^{\circ} \mathrm{C}$ or $25^{\circ} \mathrm{C}$. Thus, the

180 incarcerated DL is a transient dauer-specific phenotype that effectively results in

181 exsheathed tu391 and csu60 DL, which highlights the presence of a genetic

182 regulator in $P$. pacificus that coordinates $\mathrm{DL}$ formation with proper cuticle 183 ensheathment. Despite superficial resemblance between ensheathed wild-type DL

184 and Daf-c DL with the exsheathment defect, only the Daf-c mutations result in 185 immobilized DL, suggesting that the same genes regulate dauer entry and J2-DL 186 cuticle ensheathment in $P$. pacificus. 
Because temperature modulates the dauer decision $[55,56]$, we wondered

188 if the constitutive dauer formation phenotypes and the dauer-specific molting

189 defects are temperature-dependent. We placed ten young adult hermaphrodites

190 on OP50 at different temperatures for 5 days and scored for dauer-equivalent

191 stages. We found that for $t u 391$, lower temperature $\left(15^{\circ} \mathrm{C}\right)$ completely suppresses

192 the Daf-c phenotype, while higher temperature $\left(25^{\circ} \mathrm{C}\right)$ significantly enhances the

193 Daf-c phenotype but dramatically suppresses the dauer incarceration defect (Fig.

194 1E). $25^{\circ} \mathrm{C}$ promotes the highest proportion of free DL (92\% of third-stage larvae).

195 For csu60, lower temperature also suppresses the Daf-c phenotype, while $20^{\circ} \mathrm{C}$

196 promotes the highest proportion of incarcerated DL (9\%) (Fig. 1F). Similar to tu391

197 and other Daf-c alleles in C. elegans, free DL formation in csu60 is highest at $25^{\circ} \mathrm{C}$

$198(94 \%)$ compared to $20^{\circ} \mathrm{C}(47 \%$ of third-stage larvae) [55,57]. In contrast, wild-type

199 cultures had $100 \% \mathrm{~J} 3$ larvae at $20^{\circ} \mathrm{C}$ and $25^{\circ} \mathrm{C}$ (Table 1 ). Thus, increasing the

200 temperature resulted in higher DL formation in both alleles, while exsheathment

201 defects are most prevalent at $20^{\circ} \mathrm{C}$. Higher temperature may suppress the initiation

202 of the exsheathment defect, or it may shorten the duration of the incarceration.

203 Together, these findings indicate that the Daf-c mutants in $C$. elegans and $P$.

204 pacificus both promote dauer formation at higher temperature, but the Daf-c alleles

205 in $P$. pacificus show an additional cuticle exsheathment phenotype at $20^{\circ} \mathrm{C}$.

207 Chemosensory sensilla of Daf-c mutants

208 In C. elegans, a bilaterally symmetrical pair of anterior sensory organs known as

209 the amphid sensilla mediates chemosensory responses to water-soluble 
210 compounds and volatile odors. To determine if the Daf-c mutations affect the

211 formation or function of the amphid neurons, we used the lipophilic live dye Dil to

212 stain a subset of the amphid neurons directly exposed to the external environment.

213 Dil stains a stereotypic set of neurons by retrograde transport that appears to have

214 a modest degree of conservation across various nematode species, including $P$.

215 pacificus and C. elegans [58-60]. Moreover, several abnormal dye-filling-defective

216 mutants in C. elegans, known as the Dyf mutants, also have dauer formation

217 phenotypes; most are dauer-formation defective (Daf-d), while a few are Daf-c [61-

218 64].

219 We found that three of the twelve pairs of amphid neurons consistently stain

220 with Dil in wild-type $P$. pacificus J2, dauer, and J4 larvae, as well as young adults.

221 Based on the recent amphid neuron homology assignments between $P$. pacificus

222 and C. elegans [60], these amphid neurons correspond to the ASK, ADL, and ASH

223 neuronal homologs. Interestingly, the posterior phasmid neurons that dye-fill in all

224 developmental stages in C. elegans only show Dil fluorescence in the P. pacificus

225 DL. We found that the majority of tu391 J2 larvae (65\%) and J4/adults (97\%) stain

226 very weakly in these three pairs of amphid neurons, suggesting possible defects

227 in the differentiation or function of either the amphid neurons or the amphid sheath

228 glial cells that support them (Fig. 2A-D). In addition, $22 \%$ of $t u 391 \mathrm{DL}$ showed a

229 noticeable decrease in Dil uptake compared to $12 \%$ of wild-type DL (Fig. 2E-F).

230 Using the amphid sheath cell marker Ppa-daf-6p::Ifp [60], we found superficially

231 wild-type expression in tu391 adults; thus, the defect in dye update is likely due to

232 amphid neurons rather than glia (SI Fig. 1). In contrast to tu391, most csu60 adults 
$233(88 \%)$ take up Dil close to the rate of wildtype $(97 \%)($ SI Fig. 2). The reduction of

234 Dil uptake was the most severe in the tu391 J2 larvae and adults (weak staining

235 in $97 \%$ tu391 vs. $3 \%$ wild-type adults), but dramatically less severe in tu391 DL

236 (weak staining in only $22 \%$ tu391 vs. 12\% wild-type DL). These results suggest

237 that: (1) the amphid sensilla are remodeled during dauer development, such that

238 tu391 DL are not as dye-filling defective as tu391 J2 and adults are; (2) the two

239 Daf-c alleles have pleiotropic phenotypes related to distinct aspects of dauer entry

240 in P. pacificus.

\section{Daf-c mutations alter adult chemotaxis response}

243 Since most chemotaxis responses in non-parasitic nematodes have been based

244 on assays conducted with young adults [23-25,39], we sought to use the high

245 proportion of active Daf-c DL to examine potential behavioral changes between

246 young adults and DL. Performing chemotaxis assays on wild-type $P$. pacificus DL

247 is difficult, not only due to the paucity of dauers induced by starvation but also

248 because the cuticle surface of $P$. pacificus $\mathrm{DL}$ is covered by the long-chain

249 polyunsaturated wax ester Nematoil [35], which retards movement on the standard

250 chemotaxis medium [24,39]. Therefore, we adopted a modified agar medium

251 containing the detergent Tween, which promotes dispersal [65]. To determine if

252 Daf-c DL behave similar to wild-type DL, we tested DL of wild type, tu391, and

253 csu60 with a panel of odorants that are known attractants for $P$. pacificus adults.

254 We found that the DL of wild type and csu60, and to a lesser extent tu391, exhibited

255 strong attraction to the Oriental beetle pheromone ZTDO and the fly aggregation 
256 pheromone methyl myristate (Fig. 3A). Given this wild-type chemosensory

257 response by the Daf-c DL, we expanded the survey of DL chemosensation to the

258 other insect pheromones E-TDA, hexadecanal, and ß-caryophellene but found that

259 the Daf-c DL show no response to these odorants (SI Fig. 3) [39,66,67]. We also

260 examined the responses of Daf-c DL to a panel of C. elegans attractants: $2,3-$

261 butanedione (also called diacetyl), 2,3-pentanedione, and isoamyl alcohol (IAA)

$262[24,39]$. We found that the DL do not respond to these chemicals; $P$. pacificus

263 adults also do not respond (SI Fig. 3). Thus, the Daf-c DL exhibited near wild-type

264 responses to two known host-associated odors but have a narrower overall odor

265 response profile compared to wild-type adults. It remains to be determined the

266 degree of concordance between wild-type and Daf-c DL chemosensory profiles.

267 To better define the genetic pathways that are responsible for the

268 chemosensory differences between DL and adults, we focused on the responses

269 of the Daf-c mutants to the Oriental beetle pheromone ZTDO [68]. We observed

270 that wild-type PS312 showed the strongest difference between the DL and adults,

271 with an almost threefold stronger attraction in DL than in adults (Fig. 3B). We also

272 observed significantly stronger attraction by the adults of both Daf-c alleles, such

273 that there were no significant differences between the responses of DL and adult

274 stages. The csu60 adults were significantly more attracted to ZTDO than both wild-

275 type and tu391 adults, and despite the dye-filling defect in tu391 being more

276 pronounced in adults compared to DL, the attraction to ZTDO is stronger in tu391

277 adults than in wild-type adults. Because the passage through the dauer stage may

278 introduce lasting post-dauer changes that alter odorant receptor expression and 
279 hence modulate behavior [7,69,70], we also examined the ZTDO response of wild280 type post-dauer adults. We found that dauer passage did not result in enhanced

281 ZTDO response in adults (Fig. 3C). Thus, the enhanced ZTDO attraction in Daf-c

282 adults was not due to developmental experience, but rather the heterochronic

283 activation of a dauer-specific neuronal development.

284 To determine how the Daf-c mutants interact with chemosensory mutants

285 mediating the ZTDO response, we examined the chemotaxis behavior of the Daf-

286 c mutants in the loss-of-function obi-1(tu404) background that is defective for its

287 cGMP-dependent responses to ZTDO [68]. We found that the obi-1 background

288 did not affect ZTDO attraction in both tu391 adults and DL (Fig. 3B). In contrast,

289 the loss of obi-1 significantly reduced the ZTDO response in csu60 adults as well

290 as DL, from strong enhanced attraction to weak repulsion and neutrality,

291 respectively (Fig. 3B). We were unable to perform chemotaxis assays on the DL

292 of obi-1(tu404) mutants due to its high DL mortality rate, especially in the presence

293 of ZTDO [68]. Interestingly, while Ppa-daf-12 is epistatic to csu60 for dauer

294 formation, csu60; daf-12(tu389) double mutant adults still exhibited the csu60

295 enhanced attraction to ZTDO (Fig. 3D). Thus, the regulation of chemotaxis

296 responses can be uncoupled from dauer formation and requires a steroid hormone

297 that does not act through a DAF-12 homolog. Taken together, our results suggest

298 that tu391 acts downstream of or in parallel with obi-1, while csu60 likely acts 299 upstream of obi-1 and independent of daf-12 to mediate the enhanced pheromone 300 attraction of young adults. 
Since host odor attraction in IJs of entomopathogenic nematodes is

302 enhanced by carbon dioxide $\left(\mathrm{CO}_{2}\right)$ [21], we next tested the chemotaxis response

303 of $P$. pacificus $\mathrm{DL}$ to $\mathrm{CO}_{2}$. In $C$. elegans, well-fed adults are repelled by $\mathrm{CO}_{2}$ while

304 DL are attracted to the same concentration [20,21]. In $P$. pacificus well-fed adults,

$305 \mathrm{CO}_{2}$ also elicits a strong avoidance response which, as in $C$. elegans, is mediated

306 by a pair of internal gas sensing neurons known as the BAG neurons [20]. To

307 determine if $\mathrm{CO}_{2}$ response valence also differs between $P$. pacificus $\mathrm{DL}$ and adults,

308 we examined the response to $\mathrm{CO}_{2}$ in Daf-c DL and adults using the modified

309 chemotaxis assay. We found that wild-type $P$. pacificus DL exhibited a slightly

310 stronger avoidance response than adults, while both Daf-c DL and adults showed

311 equally robust repulsion by $\mathrm{CO}_{2}$ (Fig. 3E). Thus, similar to the enhanced response

312 to the beetle host pheromone, wild-type $P$. pacificus DL displayed a slightly

313 enhanced negative response to $\mathrm{CO}_{2}$, not a valence change. Interestingly, iL3s of

314 the mammalian parasites S. stercoralis, S. ratti, and Nippostrongylus brasiliensis

315 are also repelled by $\mathrm{CO}_{2}$ [22]. It is currently unclear how the $\mathrm{CO}_{2}$ avoidance

316 response of $P$. pacificus $\mathrm{DL}$, but not $C$. elegans $\mathrm{DL}$, factors into the different life

317 strategies of these two species in the wild.

318 Finally, to determine if genes that affect dauer development also modulate

319 olfactory behavior in other nematodes species, we also compared the odor

320 response profiles of young adults vs. DL in C. elegans [71], including the odor

321 response profiles of two well-studied Daf-c mutants, daf-2(e1370) and daf-

$3227(e 1372$, ok3125) [56,72]. We found that DL of wild-type N2 and both the Daf-c

323 mutants showed weaker odorant responses than adults. Specifically, wild-type DL 
324 showed lower attraction than adults to 2,3-butanedione, 2,3-pentanedione, and

325 IAA, consistent with the results of a previous study that included 2,3-butanedione

326 [27](Fig. 4A-C). Similar to the finding in $P$. pacificus, we also found dauer passage

327 did not result in enhanced IAA or 2,3-pentanedione attraction in post-dauer adults

328 (SI Fig. 4). Most unexpectedly, the response to IAA exhibited valence changes

329 between adults and DL: it changed from attractive to neutral in wild-type animals,

330 and from attractive to repulsive in both daf-7 mutants. To evaluate if the avoidance

331 response to IAA is due to hypersensitivity in the daf-7 DL, we also tested a 10-fold

332 lower IAA concentration (0.1\%) and found that IAA attraction was still significantly

333 reduced in both daf-7 alleles compared to wild type (Fig. 4D). Thus, C. elegans

334 wild-type and daf-2 DL showed decreased attraction compared to adults to all three

335 odorants tested, but more dramatically, daf-7 alleles showed a valence change by

336 accentuating the avoidance response of DL to IAA, suggesting that the TGF- $ß$

337 pathway is involved in both dauer regulation and IAA sensing.

339 Molecular cloning of a Daf-c mutant

340 In C. elegans, the binding of the nuclear hormone receptor DAF-12 to the steroid

341 hormones $\Delta 4$-dafachronic acids and $\Delta$ 7-dafachronic acids (DAs) restrains L2

342 larvae from undergoing the dauer fate [51]. To determine if csu60 or tu391 act

343 upstream of Ppa-daf-12, we tested a synthetic DA for its ability to ameliorate the

344 Daf-c phenotype. Previous work has shown that the constitutive dauer formation

345 phenotype of $d f c-1(t u 391)$ is rescued by feeding the worms $E$. coli OP50 containing

$3467.5 \mu \mathrm{M} \Delta 7$-dafachronic acid ( $\Delta 7$-DA) [8]. $\Delta 7$-DA has been shown to prevent dauer 
347 formation in both $P$. pacificus and the ruminant parasite Strongyloides papillosus

348 [8]. We found that both the dauer-specific exsheathment defect as well as the

349 constitutive DL phenotype of $d f c-1(t u 391)$ and csu60 were rescued by the

350 availability of $\Delta 7-\mathrm{DA}$ in the food ad libitum (Fig. 5A). These results suggest that

351 neither mutant produces the $P$. pacificus dafachronic acid that is functionally

352 equivalent to the dauer-suppressing $\Delta 7-\mathrm{DA}$.

353 To further confirm this genetic pathway for dauer regulation by epistasis

354 analysis, we constructed csu60; daf-12(tu389) double mutants and found that they

355 resembled the dauer formation defective phenotype of daf-12(tu389) [8] (Table 1),

356 consistent with the expected position of csu60 acting upstream of the nuclear

357 hormone receptor (see Methods). Similarly, the $d f c-1$ (tu391); daf-12(tu389) double

358 mutants completely masked the Daf-c phenotypes of tu391, placing tu391

359 upstream of daf-12 for the regulation of dauer formation. Hence, daf-12 is epistatic

360 to both csu60 as well as tu391. A small percentage (3.7\%) of the csu60; daf-

361 12(tu389) double mutants exhibited an ecdysis defect in the molts between late

362 larval stages (J3-J4; J4-adult), which is a synergistic phenotype not found in either

363 single mutant (Table 1). These results suggest that both mutations disrupt the

364 steroid biosynthetic and signaling pathways critical for dauer formation and 365 possibly molting.

366 Using the latest genome assembly of $P$. pacificus El Paco, we performed

367 whole genome sequencing of csu60 and identified a $\sim 95 \mathrm{~kb}$ deletion on

368 Chromosome II that contains 11 predicted protein-coding genes. Among these 369 candidates, the predicted protein sequence of PPA10139 is orthologous to the $3 \beta$ - 
370 hydroxysteroid dehydrogenase $/ \Delta 5-\Delta 4$ isomerases $(3 \beta-H S D s)$ that are key

371 steroidogenic enzymes in vertebrates (GO:0003854). PPA10139 shares 89\%

372 similarity with C. elegans HSD-2, which encodes one of three hydroxysteroid

373 dehydrogenases in C. elegans involved in the biosynthesis of dafachronic acids

374 [73]. Consistent with its potential role in repressing dauer formation, PPA10139

375 transcripts are significantly lower in DL than in the $\mathrm{J} 2, \mathrm{~J} 3, \mathrm{~J} 4$, and adult stages

376 (gene prediction Contig6-snapTAU.237) [74]. To confirm that the deletion of Ppa-

$377 h s d$ is solely responsible for the Daf-c phenotype of csu60, we made a construct

378 containing a $\sim 2 \mathrm{~kb}$ promoter of PPA10139 driving its full-length cDNA to rescue

379 the csu60 deletion by transgene complementation. Compared to the csu60 mutant,

380 the csu60 extrachromosomal transgenic rescue line showed dramatically lower

381 constitutive dauer formation (Fig. 5B). Thus, both the constitutive dauer and

382 exsheathment defects are caused by the genomic deletion containing the only

383 predicted P. pacificus hsd homolog.

384 When we investigated the possible evolutionary history of $h s d$ genes in

385 nematodes, we found only one putative ortholog of HSD-2 in each of the completed

386 genomes of the human parasites Brugia malayi and Onchocerca volvulus,

387 indicating that a single HSD is likely the ancestral state, whereas the C. elegans

388 genome contains three copies (Fig. 6A). Each hsd gene in C. elegans differs by

389 the number of transmembrane domains: $h s d-1$ encodes for two predicted domains,

$390 h s d-2$ encodes for one domain, while $h s d-3$ does not encode for any predicted

391 transmembrane domains (as predicted by SMART, which utilizes TMHMM to

392 predict transmembrane domains). Although the overall protein sequence of 
393 PPA10139 shares the highest similarity to C. elegans HSD-2, the two

394 transmembrane domains predicted for Ppa-HSD-2 argues for more functional

395 similarity to C. elegans HSD-1 (Fig. 6B).

396 We hypothesized that if Ppa-HSD-2 is the only hydroxysteroid

397 dehydrogenase in $P$. pacificus involved in dafachronic acid synthesis necessary

398 for suppressing dauer formation, then the removal of the cholesterol precursor

399 should not exacerbate the severity of the Daf-c phenotype in the presence of food.

400 In both $C$. elegans and $P$. pacificus, $5 \mu \mathrm{g} / \mathrm{ml}$ cholesterol $(\sim 13 \mu \mathrm{M})$ is routinely added

401 to NGM medium [75,76], whereas the removal of this exogenous cholesterol for

402 two generations leads to constitutive dauer formation in the presence of food [50].

403 Indeed, we observed that wild-type $P$. pacificus form $68 \%$ active $\mathrm{DL}$ at $25^{\circ} \mathrm{C}$ when

404 cultivated on NGM medium lacking the supplementary cholesterol in the medium,

405 compared to 0\% DL formation when raised with cholesterol. In contrast, cholesterol

406 removal from tu391 and csu60 cultures grown on non-cholesterol supplemented

407 NGM did not alter the percentage of DL (SI Fig. 5). The lack of response to

408 cholesterol removal suggests that Ppa-hsd-2 and the wild-type gene product of

$409 t u 391$ are responsible for most of the metabolism of cholesterol in the biosynthesis

410 of dauer-suppressing steroid hormones.

411 Utilizing whole genome sequencing of tu391 and mapping lines, as well as

412 previous fine mapping data [8], we confirmed that the mutation responsible for the

$413 d f c-1(t u 391)$ Daf-c phenotype is located on Chromosome I, which contains 17 point

414 mutations predicted to affect coding regions or splicing. To determine if the

415 mutated coding sequence is represented in the transcripts, we amplified and 
416 sequenced mixed-stage tu391 cDNA for a few candidates, including the patched-

417 related protein PPA21795 (Ppa-ptr-4), and confirmed the point mutation in the

418 transcript predicted to result in an amino acid substitution (Met>Lys). PPA21795

419 transcripts are also expressed at significantly lower levels in DL than in J2 and J3

420 larvae [74]. However, given the complexity of the PPA21795 exon-intron structure

421 (33 exons), we did not pursue transgenic rescue experiments to test if it is

422 responsible for the tu391 phenotypes.

\section{Ppa-hsd-2 influences amphid neuron identity}

425 Given the finding that $P$. pacificus HSD-2 is upstream of the putative branching

426 point for dauer formation and olfaction, we sought to investigate the possible

427 neuronal changes associated with the enhanced attraction to ZTDO in csu60 Daf-

428 c adults. Using their connectivity profiles and axon trajectories, as well as cell-

429 specific molecular markers, recent comparisons of the neuroanatomy between $C$.

430 elegans and $P$. pacificus identified homologs of the two pairs of amphid olfactory

431 neurons responsible for odor attraction, AWA and AWC [60]. The P. pacificus AWC

432 homolog, AM7, expresses Ppa-odr-7p::rfp (versus the G-protein-coding odr-3 in

433 C. elegans), whereas the AWA homolog, AM3, expresses Ppa-odr-3p::rfp (versus

434 the nuclear hormone receptor-coding odr-7 in C. elegans). We found that while

435 Ppa-odr-7p::rfp expression is unaltered in csu60 mutants (SI Fig. 6), Ppa-odr-

$4363 p::$ rfp expression is dramatically altered in csu60 in all post-hatching stages (Fig.

437 7). Specifically, in addition to the robust Ppa-odr-3p::rfp expression in AM3(AWA)

438 and the weaker expression in AM4(ASK), 68\% of csu60 J4 larvae and adults also 
439 showed unexpected expression in a third pair of amphid neurons posterior to the

440 AM3(AWA) (Table 2; Fig. 7A-B). The level of expression for each neuronal pair

441 was also stronger in csu60 neurons compared to wild type. It is not clear if the

442 expression represents a duplication of the AM3(AWA) or the AM4(ASK) cell fate.

443 To confirm that the increased Ppa-odr-3 promoter activity and ectopic cellular

444 expression correspond to increased Ppa-odr-3 mRNA transcripts in csu60, we

445 performed qPCR on mix-stage cultures and found that Ppa-odr-3 transcripts are

446 indeed $\sim 10$ fold higher in csu60 than in wildtype (SI Fig. 7).

447 To determine if the duplicated Ppa-odr-3p::Ifp expression approximated the

448 frequency of Daf-c DL at $20^{\circ} \mathrm{C}$, we also examined earlier developmental stages. In

$449 P$. pacificus, the $\mathrm{J} 1$ larva undergoes a pre-hatching larval molt inside the eggshell,

450 so the first active post-hatching stage is the $\mathrm{J} 2[77,78]$. Surprisingly, $\sim 41 \%$ of $\mathrm{J} 2$

451 larvae and $\sim 14 \%$ DL in csu60 mutants, but 0\% in wild type, also expressed ectopic

452 Ppa-odr-3p::rfp in a duplicated pair of amphid neurons (Table 2) (Fig. 7C-F). This

453 suggests that HSD activity is required to suppress a default-on Ppa-odr-3-

454 expressing cell fate prior to hatching in the $\mathrm{J} 1$ larvae or during embryogenesis. The

455 lower frequency of ectopic Ppa-odr-3p::rfp expression compared to the frequency

456 of improper dauer entry decision for csu60 may reflect the chimeric distribution of

457 the reporter gene as an extrachromosomal array during development. Due to the

458 higher RFP expression in csu60 dauers, we observed the dual ciliated dendritic 459 ends of the AM3(AWA) neurons, which was not visible in wild-type dauers [60].

460 These dendritic ends in csu60 dauers also appeared to branch posteriorly (3 out 461 of 17), which was not observed in the previous EM study on amphid dendrite 
462 morphology (Fig. 7E'-F' and SI Fig. 8) [60]. However, it is unclear whether these

463 posterior protrusions represent novel mutant dauer-stage structures or just rare

464 wild-type variants previously not visible due to lower odr-3p::rfp expression in wild-

465 type AM3(AWA). Because the duplicated Ppa-odr-3p::rfp pair was not found in

466 wild-type DL, which have enhanced attraction to ZTDO, the difference in the odr-

$4673 p:: r f p$ expression pattern alone cannot not fully explain the stronger ZTDO

468 attraction in DL compared to adults in wild type, but is consistent with the possibility

469 that the transformed wild-type amphid neuron may be involved in restricting dauer

470 entry.

471

472 Discussion

473 In order to effectively compare the olfactory behaviors of adults versus host-

474 seeking dauer larvae, we have characterized the first two Daf-c alleles in the

475 entomophilic nematode $P$. pacificus. The csu60 Daf-c phenotypes are due to a

476 deletion in the sole HydroxySteroid Dehydrogenase (HSD) in P. pacificus. This

477 gene is likely responsible for the synthesis of a $\Delta 7-D A$-like steroid ligand, which

478 regulates the conserved nuclear hormone receptor DAF-12 (Fig. 8). In C. elegans,

479 orthologous mutations in the HSD homologs on their own do not cause constitutive

480 dauer or molting defects, which suggests that steroid hormone signaling in $P$.

481 pacificus represents an ancestral state more in common with parasitic nematodes

482 than with C. elegans. Most notably, the complete loss of Ppa-hsd-2 resulted in host

483 pheromone attraction by adults in a daf-12-independent manner, and is

484 accompanied by early neuronal fate changes that lead to ectopic Ppa-odr-3 G- 
485 protein expression in a pair of chemosensory amphid neurons. By testing two well-

486 studied Daf-c mutants in C. elegans, we found that Daf-c alleles lowered the

487 already reduced wild-type DL response to adult attractants. This is in stark contrast

488 to the $P$. pacificus Daf-c alleles, which did not affect the response of DL but instead

489 increased the attraction of adults to host odorants. Thus, regulators of dauer

490 formation affect the olfactory system in developmentally distinct manners for

491 divergent species of nematodes.

493 Dafachronic acids control cuticle exsheathment and dauer entry

494 Retention of the previous cuticle, or ensheathment, is a hallmark of the infective

495 larvae of many parasitic nematodes as well as insect larvae. In insects, the pharate

496 larva forms a new cuticle while still within, but separated from, the old cuticle of the

497 previous stage [79]. In nematodes, the exsheathment of infective larvae in

498 mammalian parasites and entomopathogenic nematodes (EPN) often marks the

499 transition to the parasitic phase of the life cycle. Cues for exsheathment are a

500 combination of intrinsic physiological factors, such as the time since emergence

501 from the host, and extrinsic environmental factors, such as temperature or host

502 cues [53,80-82]. For example, in the IJs of the EPN Heterorhabditis bacteriophora,

503 exsheathing the previously attached old J2 cuticle occurs upon entering an insect

504 host, while in another EPN, Steinernema carpocapsae, IJs are unresponsive to

505 host cues for exsheathment [53]. In the pathogenic nematode of sheep,

506 Haemonchus contortus, exogenous application of an endogenous $\Delta 7-D A$

507 promotes the exsheathment of infective L3 larvae [10]. Although there are no 
508 candidate genes known to control dauer exsheathment in C. elegans- because its

509 dauers are 'naked'- conserved genes involved in molting may nevertheless

510 modulate the timing of cuticle shedding. Only one uncloned Daf-c allele, daf-

511 13(m66), has been reported to display a dauer-specific cuticle defect [83].

512 However, we could not observe either the Daf-c phenotype or the cuticle defect

513 from a revived stock. In all four C. elegans molts, the Zona-Pellucida domain

514 protein FBN-1 and integrins make up a part of the sheath between the old and new

515 cuticles [84]. We speculate that a similar mechanism may be involved in $P$.

516 pacificus such that the early detachment of the old cuticle in the Daf-c DL leads to

517 loss of contact with the mouth plug (and other orifices), resulting in the

518 incarceration of the DL inside the old J2 cuticle. Because dauer entry involves the

519 formation of a buccal cap, the retention of the J2 cuticle in $P$. pacificus DL may

520 render Daf-c DL especially prone to the incarcerated exsheathment phenotype by

521 disrupting the timing of these highly choreographed events.

\section{Steroid hormones directly regulate neuronal cell fate}

524 The ectopic expression of Ppa-odr-3p::rfp in csu60 J2 animals shows that steroid

525 hormones in $P$. pacificus are also responsible for maintaining proper cell fate in

526 putative olfactory neurons at or prior to the decision for dauer entry in J2 animals.

527 The homeotic transformation of the AWB to AWC neurons due to mutations in the

528 LIM-4 transcription factor was accompanied by changes in dendritic morphology

529 and chemotaxis behavior [85]. Based on a similar rationale, the loss of Ppa-hsd-2

530 could transform AM10(ASI) or AM6(ASG), which are normally involved in inhibiting 
531 dauer entry in C. elegans, into AM3(AWA) olfactory neurons. Coincidentally, AWA

532 and ASG are sister cells in the C. elegans cell lineage (www.wormatlas.org).

533 However, we did not observe fewer Ppa-che-1p::rfp expression in the amphid

534 neurons of Ppa-hsd-2(csu60) animals, as would be expected if one pair of the

535 neuron mis-expressing Ppa-odr-3p::rfp was derived from the AM6(ASG) or

536 AM5(ASE) neurons [60] (SI Fig. 6). Given the dynamic transcriptional profiles

537 associated with the dauer stage in $P$. pacificus [86], Daf-c mutants have the

538 potential to identify unknown molecular pathways involved in dauer commitment

539 outside of C. elegans.

541 Tributaries of DAF-12 flow into differing gene regulatory networks

542 Microarray and RNA-seq studies have shown substantial divergence of

543 dauer-specific genes between $P$. pacificus and $C$. elegans, as well as more

544 upregulated dauer-specific genes in $P$. pacificus than in $C$. elegans $[74,86]$. In $P$.

545 pacificus, the DAF-12/A7-DA module is a shared regulatory target for both dauer

546 entry and mouth form dimorphism $[37,87]$. The dye-filling defect in tu391 J2 and

547 adults, but not in the DL, suggests that defects in the amphid neurons, possibly

548 those responsible for negatively regulating dauer entry, are remodeled during the

549 dauer stage. The C. elegans daf-19 gene, which encodes the RFX family of

550 transcription factors, is required for sensory neuron cilium formation and is one of

551 the few mutations that results in both Daf-c as well as dye-filling defective

552 phenotypes [62-64]. Although dfc-1(tu391) shares the Daf-c and dye-filling

553 defective (in the non-dauers), $d f c-1$ (tu391) differs from $C$. elegans daf-19 mutants 
554 in that they do not exhibit amphid sheath defects [88] and lack a chemosensory

555 defect. Moreover, mutations in the $P$. pacificus daf-19 ortholog did not result in a

556 dauer formation defect but did show a dye-filling phenotype [47]. The particular

557 combination of phenotypes in $d f c-1(t u 391)$ has no obvious candidates.

558 In $C$. elegans, genetic dissection of the relative contribution of the

559 steroidogenic enzymes is confounded by the unknown roles the HSD-2 and HSD-

5603 paralogs play in dafachronic acid biosynthesis. HSD-1 is a component in one of

561 the two branched pathways that includes the cytochrome P450 DAF-9 required for

562 dafachronic acid biosynthesis [89]. While HSD-1 and DAF-9 use cholesterol

563 precursors to synthesize $\triangle 4-D A$, the Rieske-like oxygenases DAF-36 and DAF-9

564 synthesize $\Delta 7-\mathrm{DA}$, and together the dafachronic acid hormones converge to

565 promote reproductive development by binding to the DAF-12 receptor in the

566 inactive state $[15,73,89]$. Mutations in $h s d-1$ contribute to a nearly fully penetrant

567 Daf-c synthetic phenotype only when combined with a second mutation in NCR-

568 1/cholesterol transporter, DAF-36/Rieske-like oxygenase, or DAF-28/insulin [73].

569 In P. pacificus, the nearly fully penetrant constitutive dauer phenotype of the csu60

570 null allele at $25^{\circ} \mathrm{C}$ suggests that the absence of Ppa-hsd-2, rather than a

571 temperature sensitivity of its gene product, can lead to an almost complete de-

572 repression of dauer formation in a daf-12-dependent manner. Because the DNA

573 lesion for tu391 is not yet known, it is difficult to speculate if the tu391 mutation

574 acts in the same pathway as Ppa-hsd-2. With only a single $h s d$ in the P. pacificus

575 genome, future studies will likely reveal the degree of functional overlap the $P$. 
576 pacificus ncr-1, daf-36, and daf-9 orthologs have with Ppa-hsd-2 in dauer

577 regulation and olfactory behavior.

579 C. elegans dauers have attenuated responses to adult odors

$580 \quad$ To achieve organismal plasticity, it is has been shown that $C$. elegans dauer

581 entry involves the remodeling of glia and neural circuitry, as well as differential

582 expression of chemosensory and neuropeptide receptors $[3,4,6,7,19]$. While

583 dauer-induced remodeling of the AWC dendritic ends that increase their surface

584 area could heighten sensitivity, our results show that wild-type, daf-2, and daf-7

585 DL were actually less attracted than adults to several odors, and the DL of two daf-

5867 alleles even displayed avoidance of isoamyl alcohol. Mutation of the daf-2 gene

587 also mostly eliminated acute $\mathrm{CO}_{2}$ avoidance [20], suggesting that Daf-c mutations

588 have multiple effects on chemosensation. Other dauer-specific changes in amphid

589 gene expression and function have been observed. Two pairs of amphid neurons,

590 the AWA and AWC, are the primary mediators of chemotaxis toward attractive

591 odorants [24]. In C. elegans adults, the AWA neurons detect 2,3-butanedione

592 (diacetyl), while the AWC neurons sense isoamyl alcohol and 2,3-pentanedione.

593 A significant upregulation of neuropeptide expression during dauer entry occurs in

594 both $C$. elegans and $P$. pacificus [86], some of which may correlate with stage-

595 specific olfactory behavior, as well as dauer-specific behaviors such as nictation

596 and the change in $\mathrm{CO}_{2}$ response valence [6]. A combination of cellular identity and

597 gene expression changes may be responsible for stage-specific chemosensory

598 behaviors between adults and DL $[69,70]$. 
Our study shows that dauers in two divergent nematodes can have either

600 attenuated or enhanced odor responses relative to reproductive adults, and that

601 the differences can be intensified in the Daf-c mutants. However, the genetic

602 mechanisms coordinating these developmental and behavioral changes in these

603 two species appear to evolve in separate gene regulatory networks. Several recent

604 C. elegans studies on the remodeling that occur after entry into dauer and in post-

605 dauers adults show significant changes to olfaction and foraging behavior

$606 \quad[19,69,70]$. Our study also revealed that Ppa-hsd-2(csu60); daf-12(tu389) mutants

607 have enhanced adult odor attraction, along with a mild but noticeable ecdysis

608 defect in non-dauer larval stages. This finding invigorates the suspicion that Ppa-

609 HSD-2 may also be involved in the production of other steroid hormones, such as

610 ligands targeting the two putative $P$. pacificus ecdysone receptors [90]. Although

611 both Caenorhabditis and Pristionchus species co-occur on rotten vegetation as

612 both DL and feeding stages [91], only DL from Pristionchus species have been

613 isolated from beetles [32,34]. The robust chemoattraction to host pheromone

614 shown in this study supports the model that the DL is the preferred host-seeking

615 stage capable of locating beetle hosts. We expect we will have a better

616 understanding of how the decision for dauer entry is genetically coordinated with

617 host-associated remodeling of behavior when the cognate olfactory neurons for

618 the host pheromones are identified in $P$. pacificus. 


\section{$620 \quad$ Materials and Methods}

\section{$622 \quad$ Nematode Strains}

$623 P$. pacificus and $C$. elegans strains were maintained at $20^{\circ} \mathrm{C}$ on $\mathrm{NGM}$ plates

624 seeded with OP50 as described previously [68]. The following $P$. pacificus strains

625 were used: PS312 (California wild-type, synonymous with RS2333), Ppa-obi-

626 1(tu404) Chrl, Ppa-dfc-1(tu391) Chrl, and csu60 Chrll; Ppa-daf-12(tu389) ChrX.

627 For C. elegans, wild-type N2, as well as Daf-c mutants daf-2(e1370) and daf628 7(ok3125, e1372) were used. A previous screen for Daf-c mutants using $50 \mathrm{mM}$

629 ENU or EMS yielded two highly penetrant alleles $d f c-1$ (tu391) and Ppa-dfc630 2(tu392), but only $d f c-1(t u 391)$ was viable for further characterization [8], while 631 csu60 was isolated as an off-target mutant from a CRISPR/Cas9-mediated 632 mutagenesis against a TGF-ß ortholog (Contig115-snapTAU.31)). Each allele was 633 backcrossed three times to PS312 for the Daf-c phenotype before further

634 characterization. $\mathrm{F}_{1}$ complementation cross between dfc-1(tu391); Ppa-daf-6p::rfp

635 and csu60 did not result in RFP+ $F_{1}$ DL on food. Double mutants with obi-1(tu404)

636 were initially selected for the long body phenotype of obi-1 along with the Daf-c

637 phenotype to create rlh177 (tu391; tu404) and rlh192 (csu60; tu404). Each double

638 mutant with obi-1(tu404) was confirmed by sequencing the single nucleotide

639 deletion in tu404. The cloning and construction of the Ppa-daf-6p::rfp (tuEx231), 640 Ppa-odr-3p::rfp (tuEx265), Ppa-odr-7p::rfp(tuEx297), and Ppa-che-1p::rfp

641 (IucEx367) transgenic lines were described elsewhere [60]. To examine the 642 expression of these amphid sheath and neuronal markers in the csu60 
643 background, we crossed RFP-expressing transgenic males into csu60 J4

644 hermaphrodites, and then established individual transgenic lines from RFP+ $F_{2}$

645 progeny with the Daf-c phenotype.

$646 \quad$ For the epistasis tests, we first crossed Ppa-daf-12(tu389) hermaphrodites

647 with csu60/+ males, then isolated $F_{2}$ progeny homozygous for the csu60 deletion

648 by PCR genotyping and for the single nucleotide substitution of Ppa-daf-12(tu389)

649 by Sanger sequencing [8]. The double mutant (csu60; tu389) did not produce DL

650 on well-fed or on starved plates, indicating the Daf-d phenotype of Ppa-daf-

651 12(tu389) is epistatic to the Daf-c phenotype of csu60. Because the genetic lesion

652 responsible for tu391 was less obvious (i.e. not a large deletion), we examined the

653 segregation pattern of Daf phenotypes in addition to the genotype of the Ppa-ptr-

6544 candidate locus. We constructed a double mutant strain heterozygous for the

655 daf-12(tu389) nonsense mutation (checked by sequencing) that segregated for the

656 Daf-c phenotype in the $F_{2}$ progeny, which could be either heterozygous or

657 homozygous for the tu391 allele. We then examined the $F_{3}$ progeny for dauer

658 formation defects guided by the following rational: If the daf-12 Daf-d phenotype is

659 epistatic to tu391, then no progeny of a non-DL $F_{2}$ homozygous for daf-12(tu389)

660 will become DL. Alternatively, if the tu391 Daf-c phenotype is epistatic to daf-12,

661 then some $F_{3}$ Daf-c progeny will be homozygous for daf-12(tu389). We found that

6620 out of the $5 \mathrm{~F}_{2}$ lines from Daf-c dauers that exited were homozygous for the Ppa-

663 daf-12(tu389) genotype $\left(\chi^{2}, \mathrm{P}<0.0001\right)$, and none of the $6 \mathrm{~F}_{2}$ lines homozygous for

664 Ppa-daf-12(tu389) segregated for constitutive DL $\left(\chi^{2}, \mathrm{P}<0.0001\right)$. Furthermore, all

6656 of these daf-12(tu389) lines were Daf-d as well as homozygous for the Ppa-ptr- 
6664 mutation, suggesting that daf-12 also acts downstream of Ppa-dfc-1(tu391) for

667 dauer regulation.

668

669 Temperature and cholesterol sensitivity

670 For each experiment, 10 gravid hermaphrodites were picked onto freshly seeded

$6716 \mathrm{~cm} \mathrm{NGM}$ plates and incubated at $15^{\circ}, 20^{\circ}$, or $25^{\circ} \mathrm{C}$. Plates were scored for third-

672 stage larvae larvae (active dauer, incarcerated dauer, J3 larvae) after 5 days for

673 Daf-c or 3 days for wildtype PS312 (because they starve after 5 days under the

674 same condition). To determine how long the ensheathed DL remain incarcerated,

675 we transferred the immobilized DL at $20^{\circ} \mathrm{C}$ onto new OP50 NGM plates. After one

676 day at $20^{\circ}$ or $25^{\circ} \mathrm{C}$, these cultures were scored for the presence of active DL and

677 incarcerated DL. To determine response to the removal of cholesterol, which is

678 usually supplemented in the NGM $(1 \mu \mathrm{g} / \mathrm{ml})$, we cultivated worms on NGM lacking

679 cholesterol for three generations in the same manner before scoring for DL as

680 described above for temperature sensitivity.

681

682 Exogenous $\Delta 7$-dafachronic acid

$68310 \mu \mathrm{l}$ of $75 \mu \mathrm{M} \Delta 7$-dafachronic acid or $100 \%$ ethanol vehicle control was mixed into

$68490 \mu$ OP50 before seeding onto NGM plates. After two days, we picked 15-20

685 tu391 or csu60 gravid hermaphrodites onto each plate, which were allowed to lay

686 eggs for 5 hours before being removed for egg synchronization, or if the

687 hermaphrodites lay fewer than 50 eggs they were instead removed after 24 hours. 
688 Plates were incubated at $20^{\circ} \mathrm{C}$ or $25^{\circ} \mathrm{C}$ for $4-5$ days and then scored for dauer

689 larvae and non-dauer stages.

691 Behavioral assays

692 Pharyngeal pumping rates were observed through a Leica DM6000 upright

693 microscope with a 40x objective, without any anesthetic. J3 larvae, dauer larvae,

694 and incarcerated dauer larvae were mounted onto M9 buffer and $2 \%$ agar pads on

695 microscope slides. We counted at least four 15-second intervals per animal, which

696 were then summed to obtain pumps/minute.

697 The population chemotaxis assay for $P$. pacificus were performed on

698 covered $10 \mathrm{~cm}$ Petri dishes for $\sim 16$ hours at $22^{\circ} \mathrm{C}$ as previously described $[39,68]$.

699 However, because $P$. pacificus DL is coated with a long-chain polyunsaturated

700 wax ester (nematoil) that makes them highly hydrophobic [35], we modified the

701 chemotaxis assay by adopting a chemotaxis medium containing a detergent (10

$702 \mathrm{mM}$ MOPS pH 7; 2.5\% Tween 20; 1.5\% Bacto-agar) [65]. This MOPS/Tween

703 medium promotes both adult and DL dispersal, which when 5\% rather than 10\%

704 ZTDO was used as an attractant, likely contributed to the slightly higher $\mathrm{Cl}$ values

705 for PS312 and obi-1(tu404) adults than expected when compared to previously

706 reported [68]. DL were loaded to the center of the plate by placing and removing

707 immediately a $1 \mathrm{~cm}^{2}$ agar chunk from a culturing plate containing either wild-type

708 dauers from starved cultures or Daf-c DL from densely populated cultures. Young

709 adult worms from nearly-saturated cultures were washed twice with M9 buffer and

710 collected by centrifuging at 2500 rpm for 2 minutes. Approximately 100 worms 
711 were loaded onto each assay plate. Assays with obi-1(tu404) DL could not be

712 successfully performed due to their fragility and higher mortality. To anesthetize

713 animals at the odor sources, we place $1.5 \mu \mathrm{l}$ of $1 \mathrm{M}$ sodium azide on both sources

714 and used $100 \%$ ethanol as the counter attractant. All odorants were diluted with

715 ethanol to the following concentrations: 5\% (Z)-7-tetradece-2-one (previous

716 studies used $10 \%$ ZTDO, which resulted in more DL paralysis at loading), $1 \%$

717 methyl myristate, 1\% (E)-11-tetradecenyl acetate, 1\% (E)-11-hexadecenal, 10\%

718 ß-caryophyllene, 1\% 2,3-butadione(diacetyl), 1\% 2,3-pentadione, and 1\% isoamyl

719 alcohol. Interestingly, we found sodium azide to be superfluous when using ZTDO

720 as the attractant due to ZTDO's paralyzing effects on DL. Chemicals were

721 purchased from Fisher Scientific, Sigma-Aldrich, or Bedoukian Research

722 (Danbury, CT).

723 The chemotaxis assays for $C$. elegans were set up in a quadrant with worms

724 loaded onto the center of $6 \mathrm{~cm}$ plates containing the same MOPS/Tween agar as

725 described above, along with two opposing pairs of odors and controls [92,93]. 0.5

$726 \mu$ of $1 \mathrm{M}$ sodium azide was spotted onto each odor or counter-attractant. Since $C$.

727 elegans DL are hydrophilic, we collected DL primarily from the condensations

728 present on the underside of the lids of recently starved plates by washing the lids

729 with sterile spring water (Arrowhead, CA). For assays with Daf-c mutants, each

730 plate was scored for either DL or YA using visual confirmation (each plate can only

731 be scored for one stage). Chemotaxis assays lasted 1-1.5 hours.

732

$733 \quad \mathrm{CO}_{2}$ Chemotaxis assays 
734 Young adults were washed off plates using M9 buffer and collected in a $65 \mathrm{~mm}-$

735 Syracuse watch glass. Animals were then washed 2X with M9 buffer followed by

$7361 \mathrm{X}$ with sterile water and immediately transferred to the center of $6 \mathrm{~cm}$

737 MOPS/Tween agar plates. Excess water was removed with Whatman paper. DL

738 were directly transferred to the $6 \mathrm{~cm}$ MOPS/Tween agar plates on chunks of agar

739 from starved plates. $\mathrm{A} \mathrm{CO}_{2}$ gradient was generated by delivering specific

740 compositions of gasses through holes in the plate lids as previously described [94].

741 A certified gas mixture containing $21 \% \mathrm{O}_{2}$, balance $\mathrm{N}_{2}$ (Airgas) air control was

742 pumped through one hole whereas a certified gas mixture containing $10 \% \mathrm{CO}_{2}$,

$74321 \% \mathrm{O}_{2}$, balance $\mathrm{N}_{2}$ (Airgas) was pumped through the other hole. Gasses were

744 pumped through 1/4-inch flexible PVC tubing using a syringe pump (PHD2000,

745 Harvard Apparatus) at a flow rate of $0.5 \mathrm{ml} / \mathrm{min}$. Adults and $\mathrm{DL}$ were allowed to

746 migrate in the $\mathrm{CO}_{2}$ gradient for 1 hour and 90 minutes, respectively. Scoring

747 regions were defined as opposite halves of the assay plate $1 \mathrm{~cm}$ away from the

748 center line (as shown in the schematic). At the end of the assay, animals that

749 migrated to opposite scoring regions were scored according to the formula:

751 Chemotaxis index $(\mathrm{CI})=\left[\left(\#\right.\right.$ animals at $\mathrm{CO}_{2}$ side $)-(\#$ animals at air side $\left.)\right] /($ Total

752 \# animals at both sides).

753

754 As a control for directional bias, two assays were always run simultaneously with

$755 \mathrm{CO}_{2}$ gradients in opposite directions. Assays were discarded if the difference in 
756 the $\mathrm{Cl}$ between the two plates was $\geq 0.9$ or if fewer than 7 animals moved to both

757 scoring regions combined on either assay plate.

$759 \quad$ Dil staining

760 Bacteria were removed from worm cultures by washing with M9 buffer followed by

761 centrifuging. The cleaned worms were soaked in $300 \mu \mathrm{l} 6.7 \mathrm{mM}$ Vybrant ${ }^{\mathrm{TM}}$ Dil Cell-

762 Labeling Solution (Molecular Probes V22889) diluted in M9 with gentle inversion

763 every 30 minutes away from direct light. After 3 hours, worms were washed twice

764 with $5 x$ volume M9, followed by de-staining for at least one hour on plates seeded

765 with OP50. To isolate and stain DL, $0.1 \%$ SDS was added to the M9 used in all

766 steps. Dil staining was observed using a Leica DM6000 with a fluorescent

767 microscope. While six pairs of the amphid neurons have been observed to take up

768 Dil in wild-type PS312 [60], we scored for dye uptake in the mutants only in the

769 most robustly stained pairs- AM1, AM2, AM4 - that correspond to the C. elegans

770 neuronal homologs ASH, ADL, and ASK, respectively.

771

772 Ppa-hsd-2 transgenic rescue of csu60

773 15-20 gravid csu60 hermaphrodites with or without the Ppa-hsd-2 transgenic

774 rescue construct were picked onto plates with food, and allowed to lay eggs for 24

775 hours before removal to obtain a roughly synchronized population of embryos. The

776 plates were incubated at $20^{\circ} \mathrm{C}$ for 5 days, then scored for dauer larvae and non-

777 dauer stages, as well as for the expression of the co-injection marker Ppa-egl-

778 20p::rfp as a proxy for the presence of the Ppa-hsd-2 rescue transgene. 
779 Interestingly, we found that this method of synchronizing embryos led to a higher

780 percentage of the population remaining as DL, compared to not synchronizing the

781 embryos used in the temperature sensitivity assay.

782

783 Molecular Cloning

784 To identify the mutations, we first rough mapped the mutation to a chromosomal

785 region using Simple Sequence Length Polymorphic markers (SSLP) on 45

786 mapping lines generated with the mapping strain RS5278 (Bolivia). Each mapping

787 line was a descendent of a single $F_{2}$ dauer larva on non-starved $F 1$ plates that

788 exited when we transferred it to a fresh plate of OP50. Nematodes were washed

789 individually from each mapping line in approximate equal pellet sizes prior to

790 pooling and genomic DNA extraction using the MasterPure Kit (Epicentre). We

791 sequenced the whole genomes of $t u 391$ and csu60, as well as additional mapping

792 lines for fine mapping of tu391 and csu60, respectively [95] ( 73 million $100 \mathrm{nt}$

793 reads for each genome and bulk mapping samples; BGISEQ-500 platform with

794 DNA Nanoball technology, BGI America). For tu391, our fine mapping results with

79522 mapping lines concurred with the finding by Ogawa et al (2009) that the

796 mutation lies on Chromosome I between S563 and S440, and identified 6

797 candidate point mutations within this $\sim 2.4 \mathrm{Mbp}$ region. For csu60, rough mapping

798 indicated linkage to Chromosome II, with the additional fine mapping of 47

799 recombinant lines and the csu60 genome sequencing revealed a large $95 \mathrm{~kb}$

800 deletion on Chromosome II (1370000-1465000) that encompassed 11 open

801 reading frames. One of the predicted genes in this interval, PPA10139 (UMM-S10- 
802 1.4-mRNA-1), encodes a 376 amino acid homolog of the $C$. elegans

803 hydroxysteroid dehydrogenases 2 (HSD-2).

804 To make the Ppa-hsd-2 cDNA rescue transgene, 2063 bp of the Ppa-hsd-2

805 promoter was amplified from PS312 genomic DNA using RHL1012 and RHL1013.

806 The full-length Ppa-hsd-2 cDNA sequence was amplified from PS312 mixed stage

807 cDNA using RHL1014 and RHL1015. The Ppa-hsd-2 promoter and cDNA were

808 combined with Ppa-rpl-23 3' UTR to make pHC12. The mix injected into PS312

809 adult hermaphrodites contains the following: $2.5 \mathrm{ng} / \mu \mathrm{l} \mathrm{pHC} 12,4 \mathrm{ng} / \mu \mathrm{l} P$ pa-egl-

$81020 p:: r f p, 80 \mathrm{ng} / \mu \mathrm{l}$ csu60 gDNA (all digested with Pstl). The transgenic line was then

811 crossed into the csu60 mutant background using PCR to genotype for the deletion

812 (RHL1006 and RHL1007 will amplify 693 bp if there is a deletion; RHL1043 and

813 RHL1044 will amplify $1008 \mathrm{bp}$ if the same locus is wildtype) and scored as a

814 percentage of dauer or non-dauers (J3/J4/adult) containing the rescue transgene.

815 The csuEx54 transgene transmission rate was approximately $27 \%$.

817 Reverse transcription and Quantitative Real-Time PCR (qPCR)

818 We used two pairs of amplicons with each primer spanning over exon boundaries

819 to determine the relative level of Ppa-odr-3 transcript expression in csu60. cDNA

820 was synthesized using random hexamers (N6) and polyT primers using 500 ng

821 total RNA from mix staged worms (Thermo Fisher Maxima, M1681). We performed

822 two RNA extractions and three cDNA synthesis to run 12 technical replicates for

823 each of the primer sets. We ran the qPCR reactions using 1-2 $\mu$ CDNA in SYBR

824 Green Master Mix (Biorad) totaling $20 \mu \mathrm{l}$ in a Biorad CFX96 machine using $60^{\circ} \mathrm{C}$ 
825 as the annealing temperature. Ppa-beta-tubulin and Ppa-cdc-42 (RHO GTPase)

826 were used as housekeeping genes and the relative fold change in expression

827 between wildtype and csu60 was calculated using the delta delta $\mathrm{C}_{\mathrm{t}}$ method $828[96,97]$.

$830 \quad$ Phylogeny

831 The amino acid sequences of potential HSD homologs were first identified by

832 BLASTX searches on WormBase. The phylogeny trees were built using the entire

833 amino acid sequences by first making an alignment and removal of positions with

834 gap using T-COFFEE, followed by Maximum Likelihood phylogeny by PhyML, and

835 finally tree rendering by TreeDyn (www.phylogeny.fr)[98]. Midpoint rooting was

836 used and branch support $\geq 50 \%$ is shown.

$838 \quad$ Statistical analysis

839 Statistical tests were analyzed using GraphPad Prism 8 (La Jolla, CA) and

840 Microsoft Excel. Values were checked for normal distribution by the Kolmogorov-

841 Smirnov test before applying either multiple testing for parametric (Sidak, Tukey,

842 or Dunnett) or non-parametric datasets (Kruskal-Wallis or Mann-Whitney).

\section{Acknowledgement}

845 We thank C. Rödelsperger for whole genome sequence analysis, E. J. Corey for

$846 \Delta 7-\mathrm{DA}$ by way of the Sommer lab, J. Ly and J. Cardenas for technical assistance,

847 and the Developmental Biology class in Fall 2018 for csu60 mapping. We also 
848 thank the C. elegans Genetic Center for the daf-13(m66) strain and T. Baiocchi for

849 helpful comments on the manuscript. Funding is provided by NIH SC3GM105579

850 (to RLH), NIH 5T34GM008395-29 (to RV), NIH R01 441802-HE-31169 (to EAH),

851 and NIH F32Al147617 (to NB). 
bioRxiv preprint doi: https://doi org/10.1101/2020.03.30.016311; this version posted March 30, 2020. The copyright holder for this preprint (which was not certified by peer review) is the author/funder, who has granted bioRxiv a license to display the preprint in perpetuity. It is made available under aCC-BY 4.0 International license.

Table 1. Phenotype frequency in Daf-c mutants

\begin{tabular}{|c|c|c|c|c|c|c|c|}
\hline Strain & $\begin{array}{l}\text { Daf-c DL }{ }^{\mathrm{a}} \\
\pm \text { SEM (\%) } \\
@ 25^{\circ} \mathrm{C}\end{array}$ & $\mathbf{N}$ & $\begin{array}{l}\text { Active DL } \\
\pm \text { SEM (\%) } \\
@ 20^{\circ} \mathrm{C} \\
\end{array}$ & $\begin{array}{l}\text { Mlt defect } \\
\pm \text { SEM (\%) } \\
@ 20^{\circ} \mathrm{C} \\
\end{array}$ & $\mathbf{N}$ & $\begin{array}{l}\text { Daf-c } \mathrm{DL}^{\mathrm{a}} \\
\pm \mathrm{SEM} \\
(\%) @ 15^{\circ} \mathrm{C}\end{array}$ & $\mathbf{N}$ \\
\hline Wild-type PS312 & 0 & $512(3)$ & 0 & 0 & $482(3)$ & ND & \\
\hline hsd-2(csu60) & $99 \pm 0.4$ & $1577(5)$ & $47 \pm 2$ & $9 \pm 1.2^{b}$ & $1891(5)$ & $2 \pm 0.4$ & $1250(5)$ \\
\hline hsd-2(csu60); daf-12(tu389) & 0 & $1172(6)$ & 0 & $3.7 \pm 3.4^{c}$ & $808(6)$ & ND & \\
\hline daf-12(tu389) & 0 & $1250(7)$ & 0 & 0 & $950(7)$ & ND & \\
\hline $\begin{array}{l}\text { hsd-2(csu60); csuEx54[Ppa- } \\
\text { hsd-2p::hsd-2 cDNA] }\end{array}$ & ND & & $11 \pm 2.5$ & 0 & $2388(6)$ & ND & \\
\hline$h s d-2(\operatorname{csu} 60)+\Delta 7-\mathrm{DA}$ & ND & & 0 & 0 & $284(3)$ & ND & \\
\hline dfc-1(tu391) & $96 \pm 0.9$ & $1520(7)$ & $28 \pm 3.5$ & $25 \pm 2.6^{b}$ & $1742(11)$ & 0 & $577(7)$ \\
\hline dfc-1(tu391); daf-12(tu389) & 0 & $1005(7)$ & 0 & 0 & $1003(7)$ & ND & \\
\hline$d f c-1(t u 391)+\Delta 7-D A$ & 0 & $426(4)$ & ND & ND & & ND & \\
\hline
\end{tabular}

Values based on the progeny of 10 gravid hermaphrodites scored 5 or 6 days later.

ancludes both active and exsheathment defective incarcerated dauer larvae formed under reproductive conditions among all J3equivalent larvae.

${ }^{\mathrm{b}}$ Transient exsheathment defective Daf-c dauers; no molting defect was found in non-dauers.

'Transient molting defect in J3-J4 or J4-Adult molts.

$\mathrm{N}$ : sample size with the number of experiments is given in parentheses.

ND: not determined

SEM: standard error of the mean 
bioRxiv preprint doi: https://doi.org/10.1101/2020.03.30.016311; this version posted March 30,2020. The copyright holder for this preprint (which was not certified by peer review) is the author/funder, who has granted bioRxiv a license to display the preprint in perpetuity. It is made available under aCC-BY 4.0 International license.

Table 2. Ppa-odr-3p::rfp expression in Ppa-hsd-2(csu60)

\begin{tabular}{ccccc}
\hline \hline Strain & Stage & $\begin{array}{c}\text { No. Expression two pairs } \\
\text { AM3(AWA) + AM4(ASK) }\end{array}$ & $\begin{array}{c}\text { No. Expression three pairs } \\
\text { AM3(AWA) + AM4(ASK) + } \\
\text { unknown (\%) }\end{array}$ & N \\
\hline PS312 wildtype & J2 & 10 & $0(0 \%)$ & 10 \\
& Dauer & 22 & $0(0 \%)$ & 22 \\
Ppa-hsd-2(csu60) & J4ladult* $^{*}$ & 17 & $0(0 \%)$ & 17 \\
& $\mathrm{J2}^{* *}$ & 17 & $12(41 \%)$ & 29 \\
& Dauer & 24 & $4(14 \%)$ & 28 \\
\hline \hline
\end{tabular}

An additional 22 adults with the same expression pattern were observed in Hong et al, eLife, 2019

*Including some partial dauers that had darkened intestines but did not have buccal caps and radial constriction.

$\mathrm{N}$ : sample size 
Table 3: Primers

\begin{tabular}{|c|c|c|}
\hline Primer & Sequence $\left(5^{\prime} \rightarrow 3^{\prime}\right)$ & Target and Purpose \\
\hline RHL940 & GAGTTCCCAGAGATGTCTG & Ppa-ptr-4 cDNA \\
\hline RHL942 & GATCCACGAGATAACCTTG & Ppa-ptr-4 cDNA \\
\hline RHL941 & CAAAGACATTGGGAATGTAATC & Sequencing primer for Ppa-ptr-4 cDNA \\
\hline RHL987 & ATTGTTATTGTTGATTGTTACG & $\begin{array}{l}\text { Gibson Assembly vector backbone containing } \\
\text { Ppa-rpl-23 3'UTR from pHC5.4 for Ppa-ptr-4 and } \\
\text { Ppa-hsd-2 rescue constructs. }\end{array}$ \\
\hline RHL988 & TCTTTCTAGAAGATCTCCTAC & $\begin{array}{l}\text { Gibson Assembly vector backbone containing } \\
\text { Ppa-rpl-23 3'UTR from pHC5.4 for Ppa-ptr-4 and } \\
\text { Ppa-hsd-2 rescue constructs. }\end{array}$ \\
\hline RHL927 & AGGGAAATGCAGCGCGAG & Ppa-hsd-2 cDNA \\
\hline RHL930 & GAGGCACGAGCAACTTGG & Ppa-hsd-2 cDNA \\
\hline RHL1006 & TTCTCCTTCAGGCAAACTCGTAG & Genotyping the csu60 deletion \\
\hline RHL1007 & TACGGTATCGCGGTTGGGTATCG & Genotyping the csu60 deletion \\
\hline RHL1012 & $\begin{array}{l}\text { TAGGAGATCTTCTAGAAAGAACAA } \\
\text { GCTCACTAACCAGTG }\end{array}$ & Ppa-hsd-2 promoter for rescue construct \\
\hline RHL1013 & $\begin{array}{l}\text { GTTGGTGCATTTTTACTAGAATTTA } \\
\text { CGATAGGAAATAG }\end{array}$ & Ppa-hsd-2 promoter for rescue construct \\
\hline RHL1014 & $\begin{array}{l}\text { TCTAGTAAAAATGCACCAACGCTG } \\
\text { CTGC }\end{array}$ & Ppa-hsd-2 cDNA for rescue construct \\
\hline RHL1015 & $\begin{array}{l}\text { TAACAATCAACAATAACAATTCATT } \\
\text { TGAAGTAGAAAAACAACACAGCC }\end{array}$ & Ppa-hsd-2 cDNA for rescue construct \\
\hline RHL1043 & GTTCAACCAGTGAAATCGCATTC & $\begin{array}{l}\text { Genotyping the csu60 deletion for identification of } \\
\text { wild-type genotype }\end{array}$ \\
\hline RHL1044 & GCTTCTGAATAGATTGTCTGACG & $\begin{array}{l}\text { Genotyping the csu } 60 \text { deletion for identification of } \\
\text { wild-type genotype }\end{array}$ \\
\hline RHL1114 & GTAGTGACAAAGCGATGGAC & Genotyping for the tu391 mutation in Ppa-ptr-4 \\
\hline RHL1115 & AGTCATACGACTGCAGACTG & Genotyping for the tu391 mutation in Ppa-ptr-4 \\
\hline
\end{tabular}




\begin{tabular}{|l|l|l|}
\hline RHL1116 & CCAATAAGTCTATCTCAGAGCC & Genotyping for the exon 2 mutation in Ppa-ptr-4 \\
\hline RHL1117 & GAGGGACTGAACATTTTCATTAC & Genotyping for the exon 2 mutation in Ppa-ptr-4 \\
\hline RHL1111 & TTACTCCATAGGAAAGGACC & $\begin{array}{l}\text { Ppa-ptr-4 crRNA for CRISPR/Cas9 genome } \\
\text { editing in exon } 2 \text { (csu77) }\end{array}$ \\
\hline RHL1110 & ACATCAACATGATCGTTGCG & $\begin{array}{l}\text { Ppa-ptr-4 crRNA for CRISPR/Cas9 genome } \\
\text { editing in exon 12 (tu391) }\end{array}$ \\
\hline RHL1097 & GTTGATAAGGAACAACAGCAGCG & Ppa-odr-3 qPCR primer for exon 2 \\
\hline RHL1098 & CCGCATTCTCCAGCTCCTAAC & Ppa-odr-3 qPCR primer for exon 2 \\
\hline RHL1099 & $\begin{array}{l}\text { GAATCGTTTTCAATAACACGGTCA } \\
\text { C }\end{array}$ & Ppa-odr-3 qPCR primer for exon 5 \\
\hline RHL1100 & AATCATAGCTTTCTCGGGCTCC & Ppa-odr-3 qPCR primer for exon 5 \\
\hline
\end{tabular}




\section{Figures}

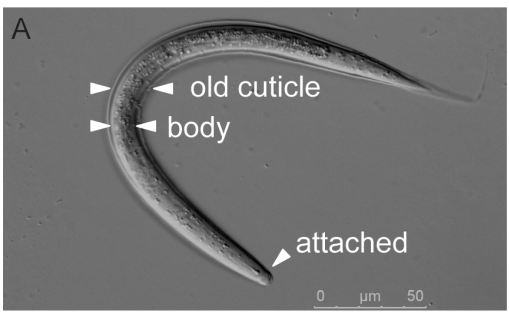

D

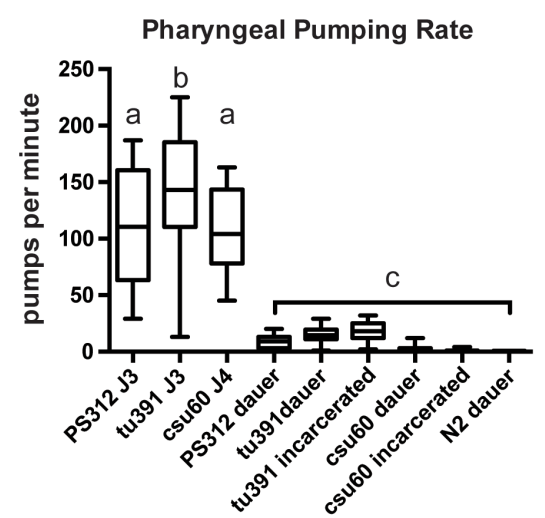

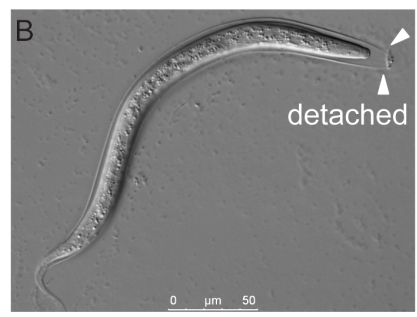

$\mathrm{E}$
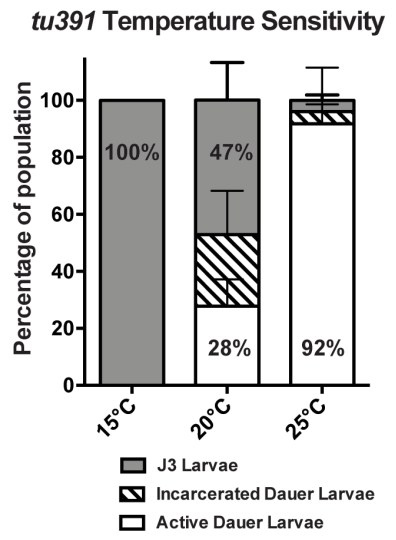

C

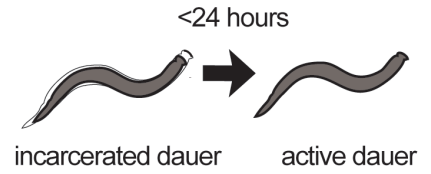

$\mathrm{F}$
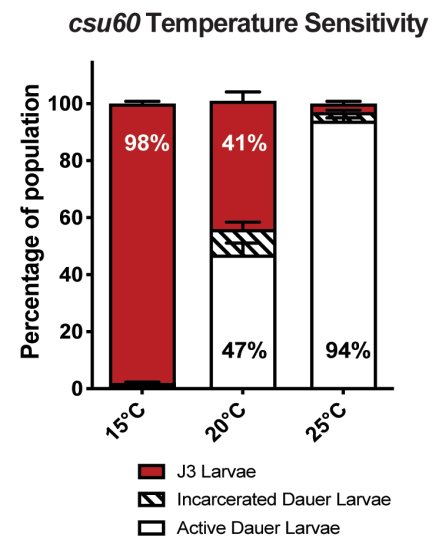

Figure 1. Incarcerated larvae are temperature-dependent constitutive dauer larvae. Ten young adult hermaphrodites on food were transferred to different temperatures for 5 days, and their progeny were then scored for dauer-equivalent J3 stages. (A) Ensheathed wild-type PS312 dauer larvae. The effete J2 cuticle surrounding the body is visible. (B) In an incarcerated tu391 dauer larva, the J2 cuticle is not attached at the head in nearly half of tu391 constitutive dauer larvae. (C) The illustration depicts that the incarceration is transient. (D) Incarcerated dauer larvae $\left(n_{\text {tu391 }}=22 ; n_{\text {csu60 }}=30\right)$ exhibit pharyngeal pumping rates similar to active mutant dauer $\left(\mathrm{n}_{\mathrm{tu} 391}=22 ; \mathrm{n}_{\mathrm{csu} 60}=13\right)$ and wild-type $P$. pacificus and $C$. elegans dauer $\left(\mathrm{n}_{\mathrm{Ppa}}=24 ; \mathrm{n}_{\mathrm{Pel}}=18\right)$, which are significantly different from that of the J3 and J4 larval stages (PS312 J3 n=32; tu391 J3 n=40; csu60 J4 n=13) (letters denote significant differences at $P<0.001$, One-way ANOVA, Tukey's Multiple Comparisons test). (E) The proportions of tu391 J3 larvae, active dauer larvae, and dauer larvae differ at $15^{\circ} \mathrm{C}(n=577), 20^{\circ} \mathrm{C}(n=2091)$, and $25^{\circ} \mathrm{C}(n=1622)$. Incarcerated dauer larvae are $25 \%$ of all third-stage larvae larval stage animals at $20^{\circ} \mathrm{C}$. Wild-type PS312 are $100 \% \mathrm{~J} 3$ at all temperatures. (F) The proportions of csu60 J3 larvae, active dauer larvae, and incarcerated dauer larvae differ at $15^{\circ} \mathrm{C}$ $(n=1250), 20^{\circ} \mathrm{C}(n=1891)$, and $25^{\circ} \mathrm{C}(n=1466)$. Incarcerated dauer larvae are $9 \%$ of all third-stage larvae larval stage animals at $20^{\circ} \mathrm{C}$. Additional details are found in Table 1. 


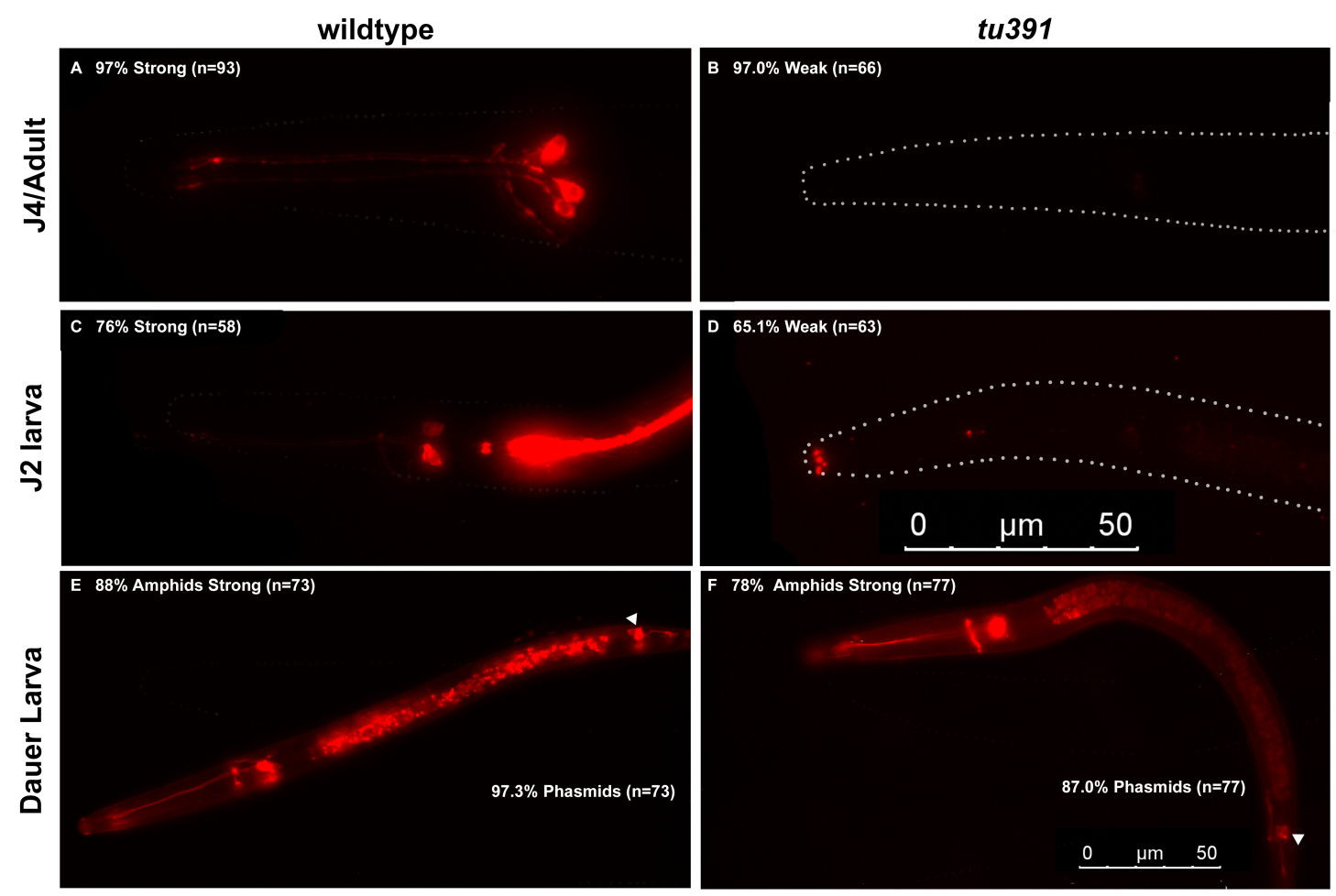

Figure 2. tu391 is defective in Dil uptake. (A-D) Adult, J4, and J2 tu391 larvae show significantly weaker staining in the amphid neurons when compared to wildtype PS312 animals. (E-F) However, the tu391 dauer larvae take up dye at a level comparable to wild type, including in the posterior phasmid neurons that take up dye only in the DL stage (triangle). Worms were exposed to $6.7 \mathrm{nM}$ Dil in M9 for 3 hours and scored categorically based on dye intensity and the number of visible neurons. $0.1 \%$ SDS with M9 was used for staining dauer larvae. Max projections of stacked images, exposure $200 \mathrm{~ms}$ (A, B) or $524 \mathrm{~ms}(\mathrm{C}-\mathrm{F})$. Anterior is left and dorsal is up. The $50 \mu \mathrm{m}$ scale bar in (D) represents (A-D), and the scale bar in (F) represents $(\mathrm{E}-\mathrm{F})$. 
A

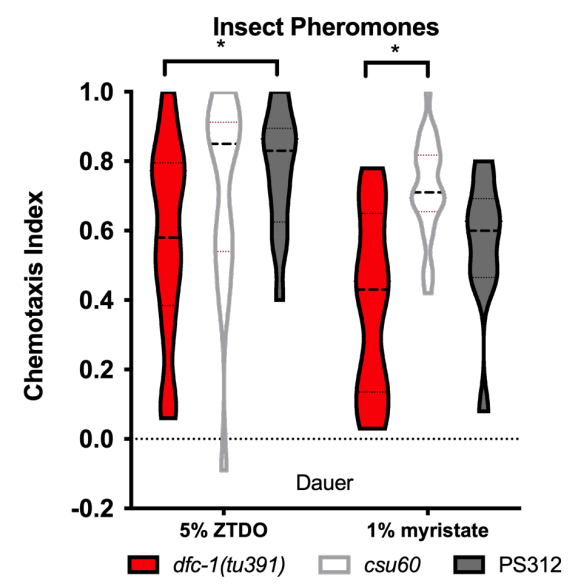

B

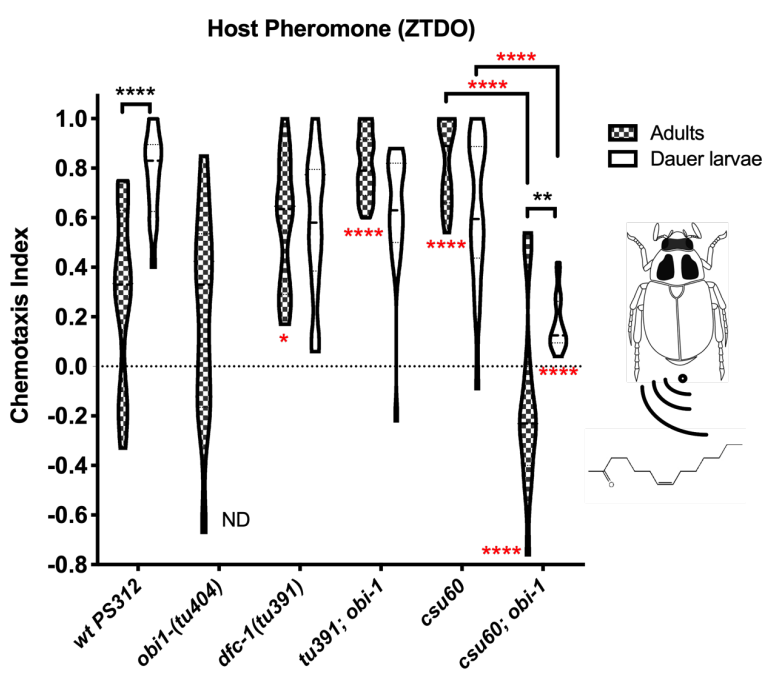

C

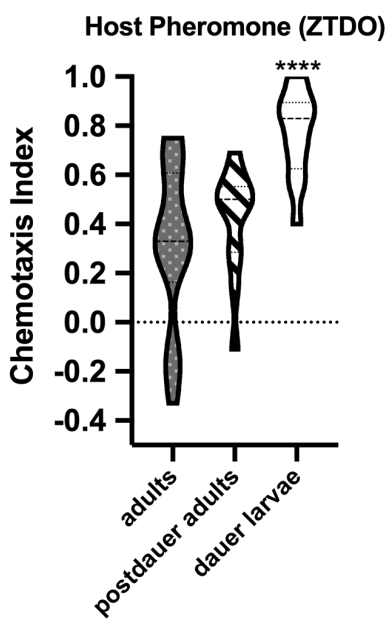

D

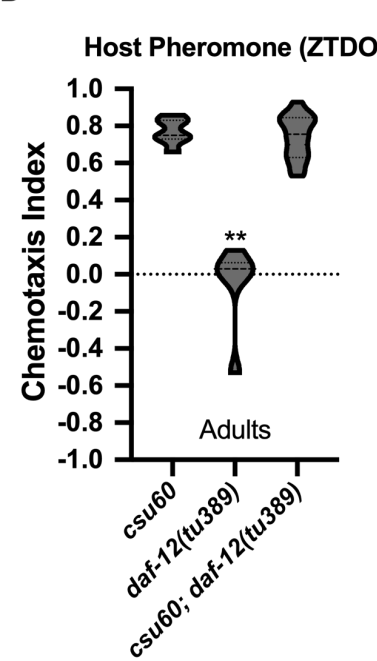

E

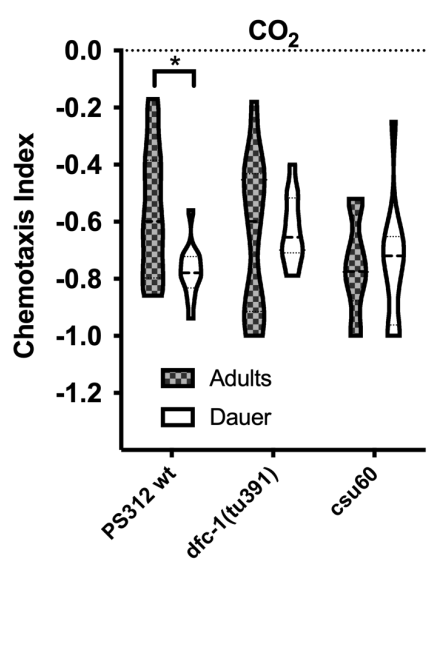

Figure 3. Olfactory responses of $\boldsymbol{P}$. pacificus dauer larvae. (A) Dauer larvae of wild-type and Daf-c mutants show robust attraction to $5 \%$ Oriental beetle pheromone z-(7)-tetradecen-2-one (ZTDO) and 1\% house fly aggregation pheromone methyl myristate. Two-way ANOVA with Dunnett's multiple comparisons test to wildtype. (B) Daf-c mutations enhance adult attraction to the host beetle pheromone ZTDO in tu391 and csu60, while the loss of obi-1 significantly reduces attraction csu60 attraction to ZTDO. Two-way ANOVA for differences by genotype with Tukey's multiple comparisons test in red asterisks; asterisks below show differences to PS312. Black asterisks denote differences between adults and dauer by Sidak's test. (C) Post-dauer adults show a similar response to ZTDO as the never-dauer adults. One-way ANOVA against adults with Dunnett's post-hoc test. (D) The steroid hormone receptor encoded by Ppadaf-12 is not required for the enhanced attraction to ZTDO in adult csu60. Oneway ANOVA against csu60 with Kruskal-Wallis test. (E) The response to carbon dioxide differs slightly between wild-type adults and dauers, but does not differ 
between adults and dauer larvae in the Daf-c mutants. Two-way ANOVA between adults and dauer with Sidak's test. For each category, 12-15 assays were performed over three trials. Violin plots show median with quartiles. ${ }^{*} P<0.05$; ${ }^{* *} P<0.01 ;{ }^{* * *} P<0.0001$. 
A

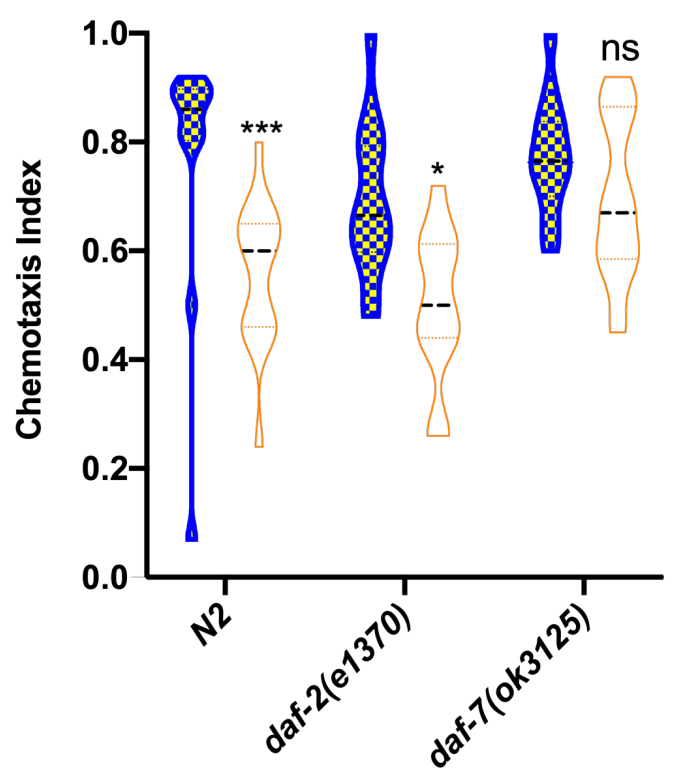

C

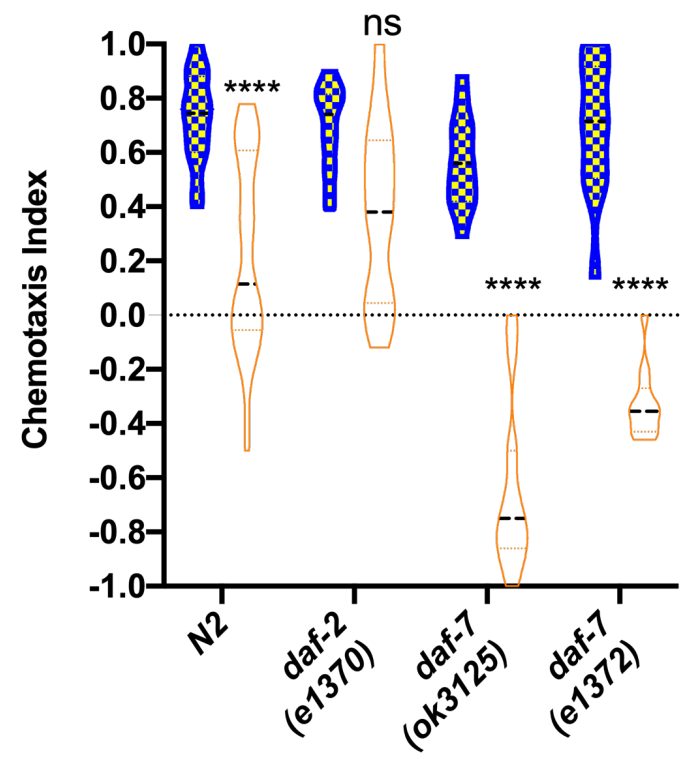

B

$1 \%$ 2,3-Pentanedione

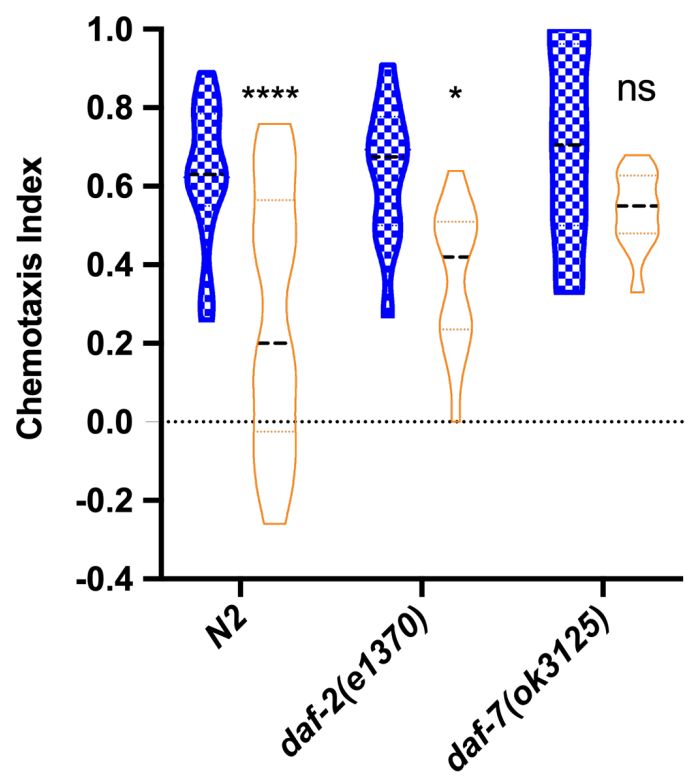

D

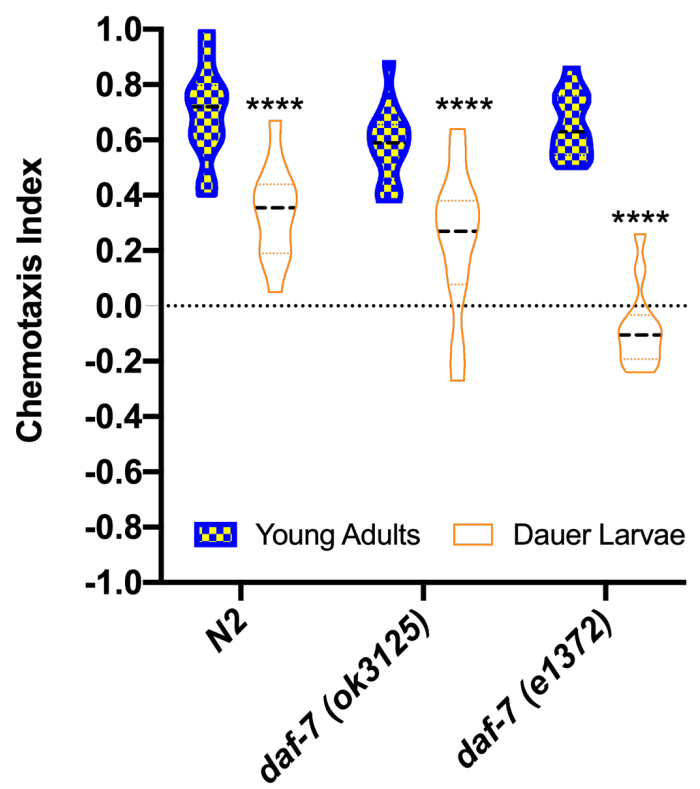

Figure 4. Olfactory responses of $C$. elegans dauer larvae. Chemotaxis between adults and dauer larvae differ significantly in response to (A) 1\% 2,3-butanedione, (B) $1 \%$ 2,3-pentanedione, and (C-D) $1 \%$ and $0.1 \%$ isoamyl alcohol in wild-type N2 and the Daf-c mutants daf-2 and daf-7. For A and C: One-way ANOVA between adults and dauer with Kruskal-Wallis test. For B and D: Two-way ANOVA between adults and dauer with Sidak's multiple comparisons test. ${ }^{*} P<0.05,{ }^{* *} P<0.01$, ${ }^{* * * \star} P<0.0001$, ns: not significant. For each category, 12 assays were performed over $>3$ trials. Violin plots show medians with quartiles. 
A

\section{$\Delta 7$-DA rescue}

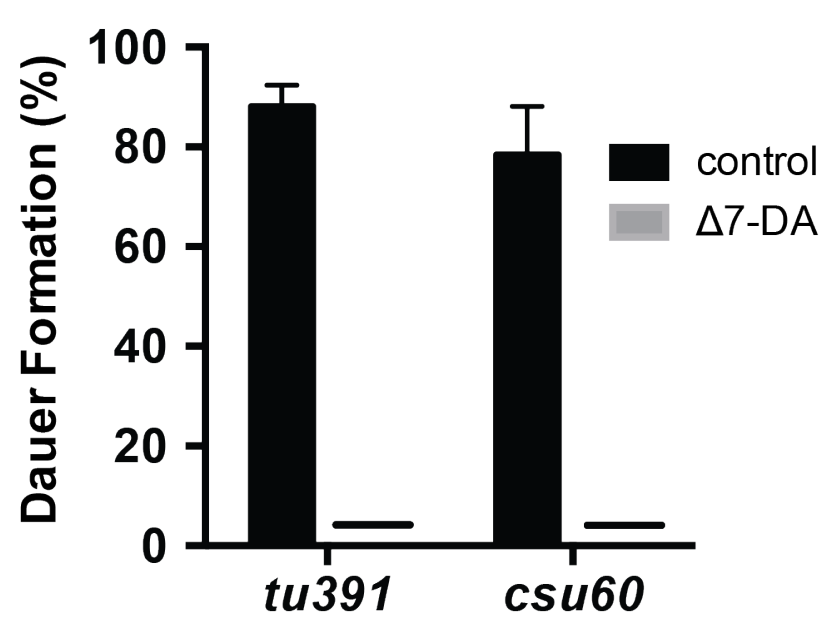

B Ppa-hsd-2 rescue

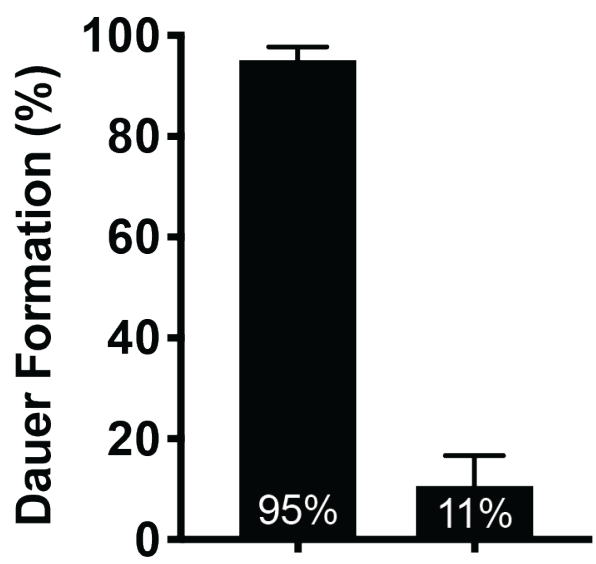

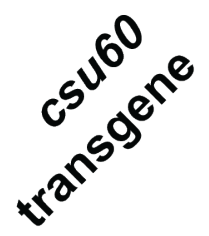

Figure 5. Hormonal and transgenic rescue of Daf-c mutants. (A) The constitutive dauer phenotype in both Daf-c mutant alleles is completely rescued by exogenous $\Delta 7$-dafachronic acid but not by the ethanol control. (T-test between mock and DA treated. $P<0.0001$. Sample size: $t u 391$ control $=435$; tu391 treated $=426$; $\operatorname{csu} 60$ control $=210$; csu60 treated $=284$ ). $(\mathrm{B}) \mathrm{A}$ transgene carrying the Ppa-hsd-2 promoter driving its own cDNA strongly rescues the constitutive dauer defect in csu60 (T-test between transgenic and non-transgenic populations. $P<0.0001$; Sample size: $c s u 60=2246$; csu60 segregating for the rescue transgene $=2388$ ). Dauer formation is the percentage of DL in all third-stage larvae or older. 


\section{A}

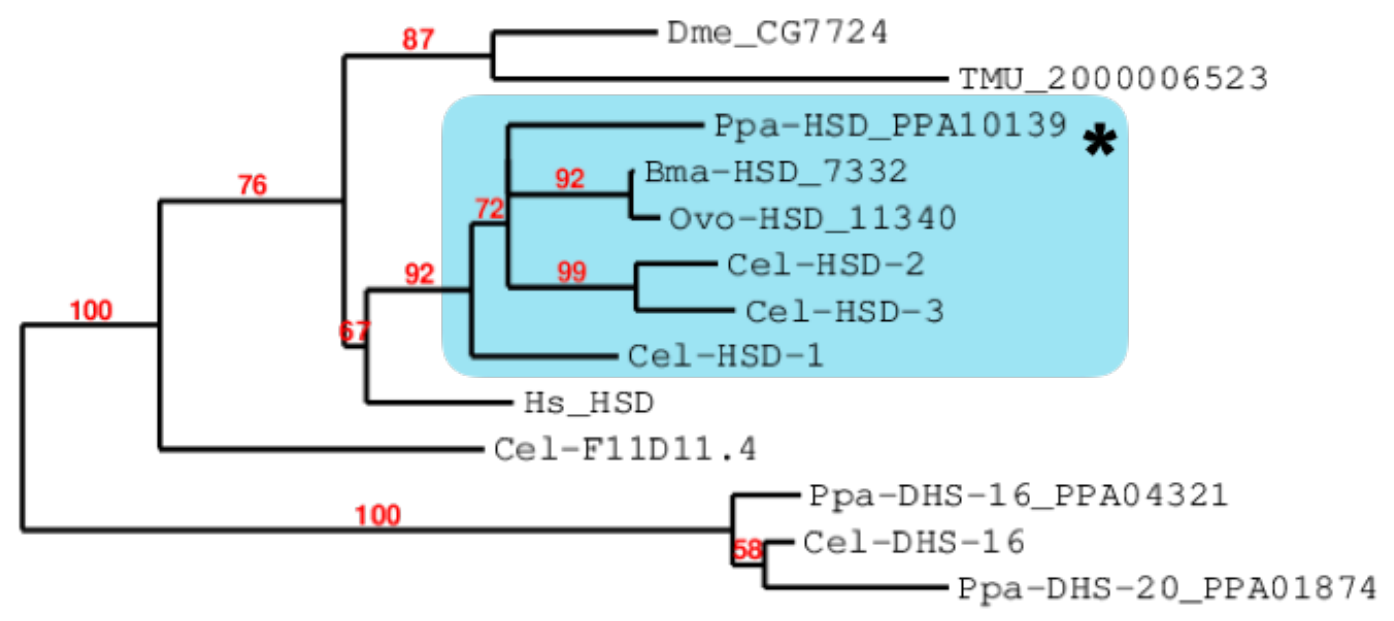

1.

B

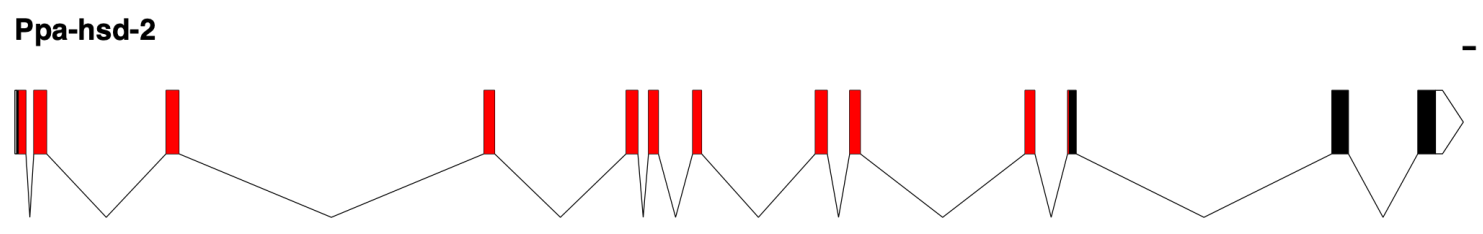

Figure 6. Ppa-hsd-2 phylogeny and gene annotation. (A) Orthology assignment for Ppa-HSD-2 (PPA10139). Maximum Likelihood phylogeny trees were generated by PhyML (phylogeny.fr). Midpoint rooting was used and only branch support $\geq 50 \%$ is shown. Protein sequences for $P$. pacificus are "Ppa-" (orthology same as Wormbase.org) and for C. elegans "Cel-". Sequences from additional species are Drosophila melanogaster (Dme), Onchocerca volvulus (Ovo), Brugia malayi (Bma), Trichuris muris (Tme), and Homo sapiens (Hs). Dehydrogenases DHS-16 from C. elegans along with DHS-16 and DHS-20 from $P$. pacificus are closest to the HSDs and are used as an outgroup. (B) Ppa-hsd-2 gene annotation (PPA10139). Red denotes the predicted 3 beta-hydroxysteroid dehydrogenase protein family spanning amino acid residues 6-274 (exons 1-11). Gene annotation was created using the Exon-Intron Graphic Maker on wormweb.org, and the SMART webtool was used to predict protein domains (smart.embl-heidelberg.de Letunic et al., 2014 and 2017). Unfilled blocks are 5' and 3' UTRs. Scale bar: 100 bp. 

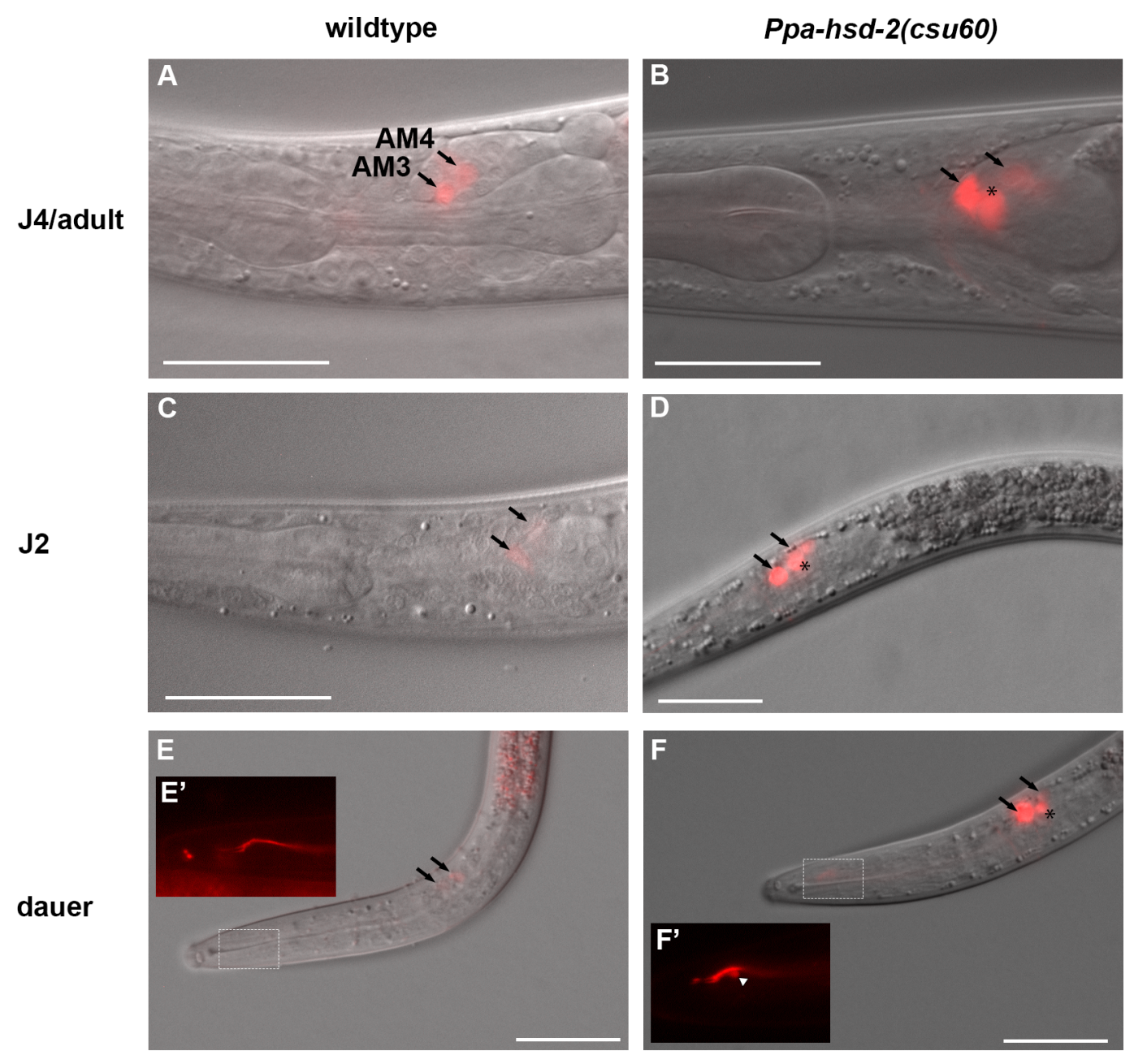

Figure 7. Ectopic expression of the Ppa-odr-3p::rfp reporter in amphid neurons in Ppa-hsd-2(csu60). (A-B) Representative DIC and fluorescent overlaid images of $\mathrm{J} 4$ and adults. In wildtype, Ppa-odr-3p::rfp expression is found in AM3(AWA) and AM4(ASK) amphid neurons (arrows). In csu60 mutants, reporter expression in AM3(AWA) and AM4(ASK) is stronger than wildtype and is found in an additional pair of amphid neurons posterior to the AM3(AWA)(*). (C-D) Ectopic Ppa-odr-3p:::ffp expression is also found in csu60 J2 larvae. (E-F) Ppa-odr-3p:::rfp expression is also found in csu60 DL. (E') RFP fluorescence shows dual ciliated dendritic ending outlined in the dotted rectangular area of a different DL. (F') RFP fluorescence shows dual ciliated dendritic ending outlined in the dotted rectangular area of the same DL. Triangle points to a type of posterior protrusion observed in 3 out of 17 csu60 DL but not in wild-type DL (additional examples are shown in SI Fig. 8). Images of wild-type samples were taken at $700 \mathrm{~ms}$ exposure, and csu60 samples were taken at $700 \mathrm{~ms}$ exposure or lower due to much stronger expression level. Sample sizes are shown in Table 2. Anterior is left and dorsal is up. Scale bar: $25 \mu \mathrm{m}$. 


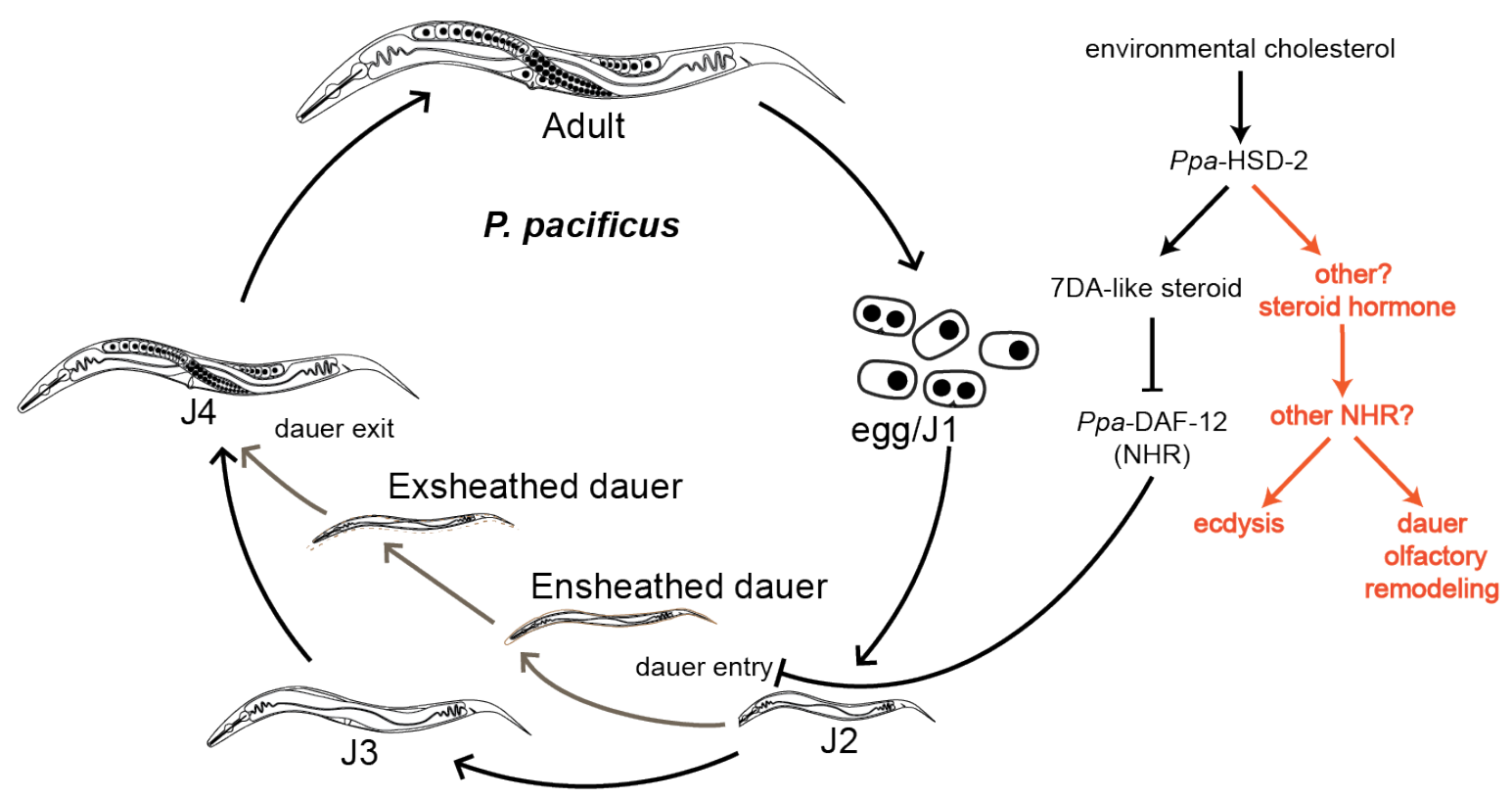

Figure 8. A model for the multiple roles of Ppa-HSD-2 in coordinating dauer entry, molting, and olfaction. From the cholesterol precursor, Ppa-HSD-2 is required for the synthesis of a $\Delta 7$-dafachronic acid-like steroid hormone (7DA). Binding of $\triangle 7$-DA to the DAF-12 nuclear hormone receptor represses decision for dauer entry. Ppa-HSD-2 may also be required for producing other steroid hormones that can explain the DAF-12-independent phenotypes of defective ecdysis during reproductive development and enhanced host odor attraction in the Daf-c adults. 


\section{Supplementary Figures}
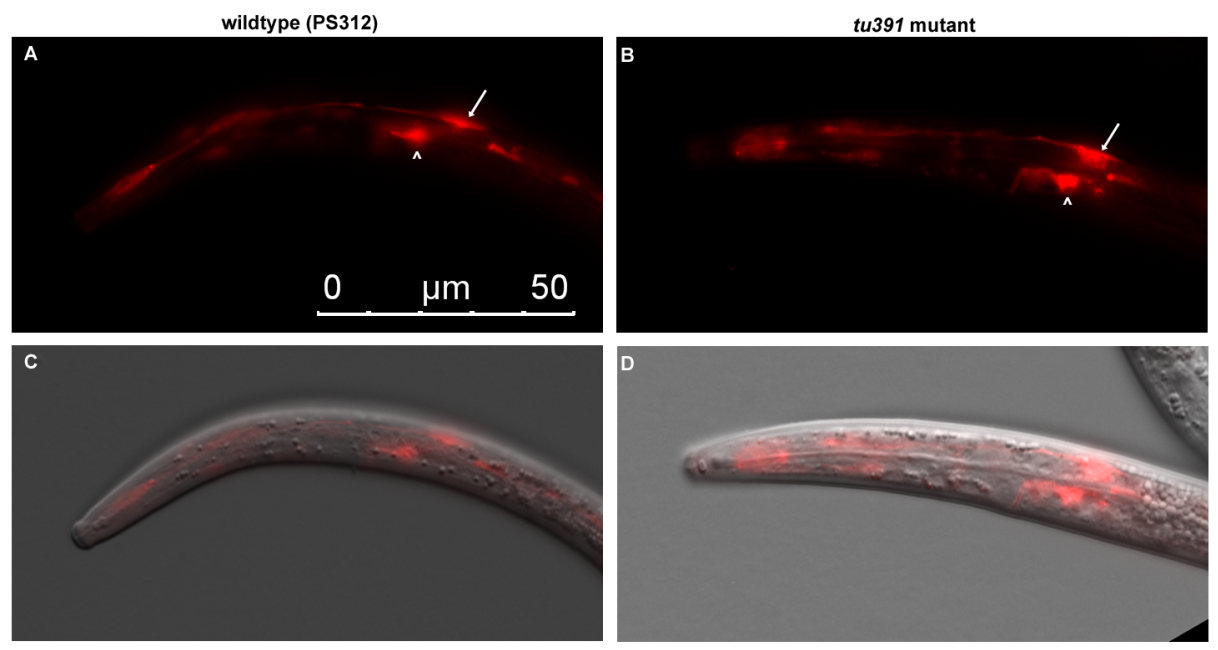

SI Figure 1. tu391 dauer larvae have normal amphid sheath morphology. Fluorescent and DIC overlay images of wild-type PS312 (A, C) and tu391 (B, D) dauers show similar expression profiles of the amphid sheath marker Ppa-daf$6 p::$ ifp (RS2818) indicated with arrow heads. "^" marks the excretory cell. Anterior is left and dorsal is up. The scale bar in A represents all panels.
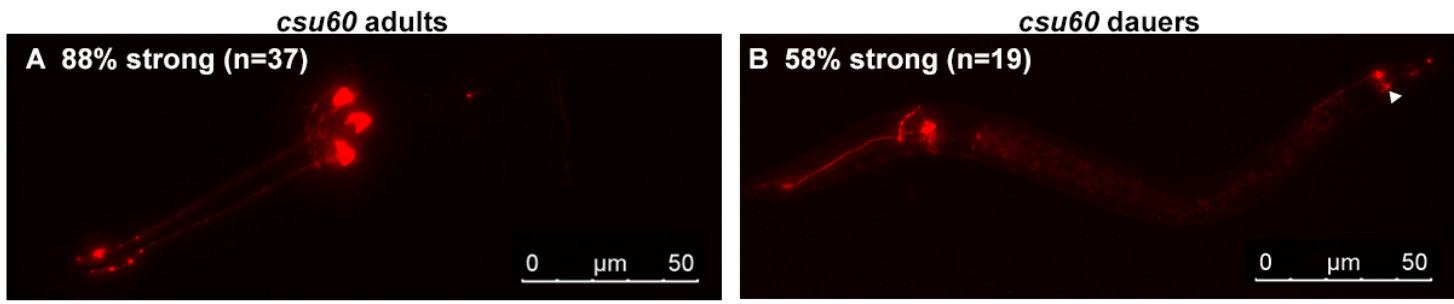

SI Figure 2. csu60 is wild type in live dye Dil uptake. (A) Adult and (B) dauer larvae take up dye at a level comparable to wildtype (Fig. 2). Max projections of stacked images, exposure $200 \mathrm{~ms}$. 
A

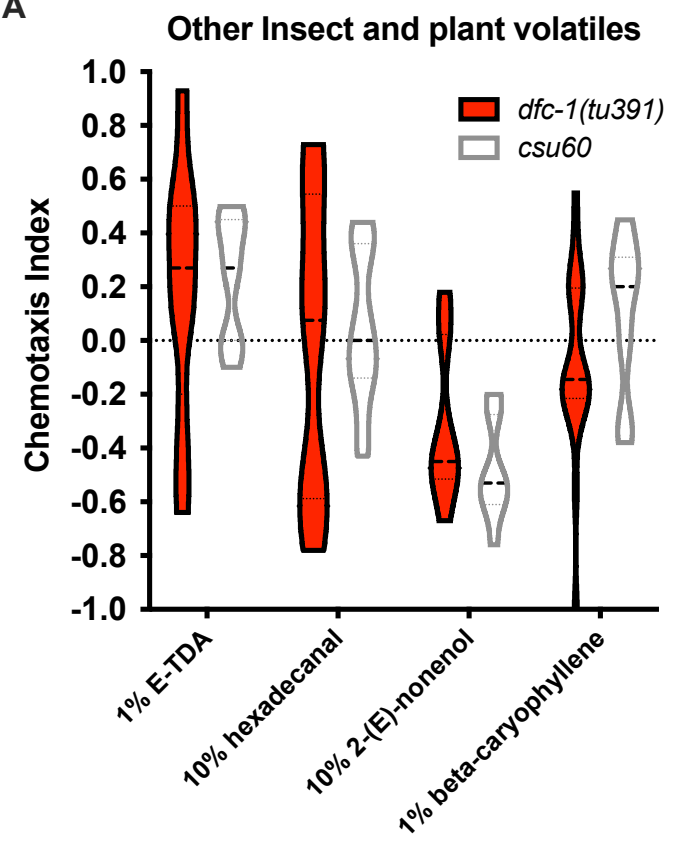

B

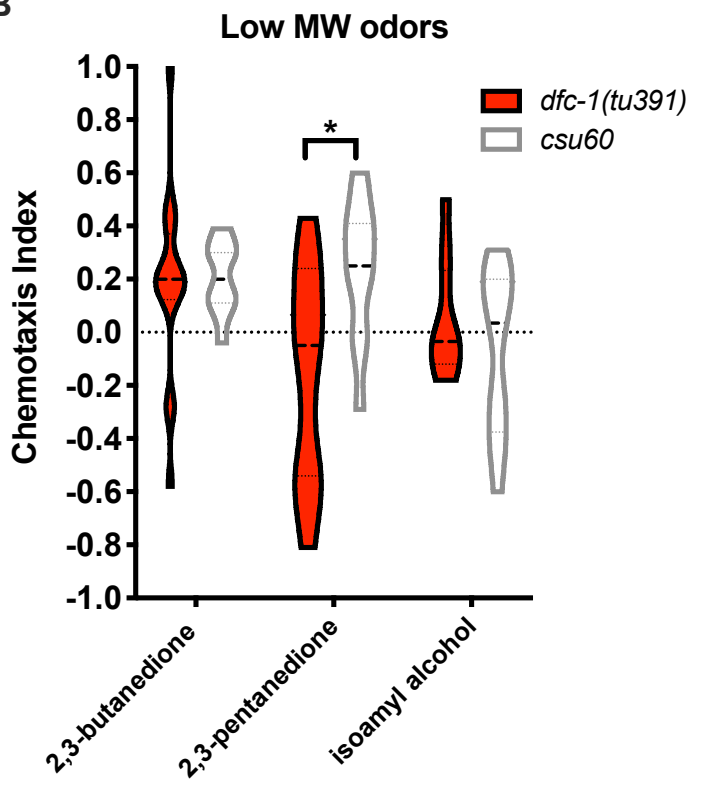

SI Figure 3. Olfactory responses of Daf-c dauer larvae. (A) Dauer larvae of DafC mutants do not respond to other insect pheromones known to be attractive to adults (1\% E-11-tetradecenyl acetate (E-TDA), 10\% hexadecanal, 10\% 2-(E)nonenol) and the plant defense volatile $1 \%$ ß-caryophyllene (Hong et al, Current Biology, 2006). (B) Dauer larvae of Daf-c mutants also do not respond to small molecular weight compounds that are strong attractants to C. elegans: $1 \% 2,3-$ butanedione (diacetyl), 1\% 2,3-pentanedione, and 1\% isoamyl alcohol. Two-way ANOVA with Sidak's multiple comparisons test between tu391 and csu60. Violin plots show medians with quartiles. ${ }^{*} P<0.05$. 


\section{C. elegans post-dauer}

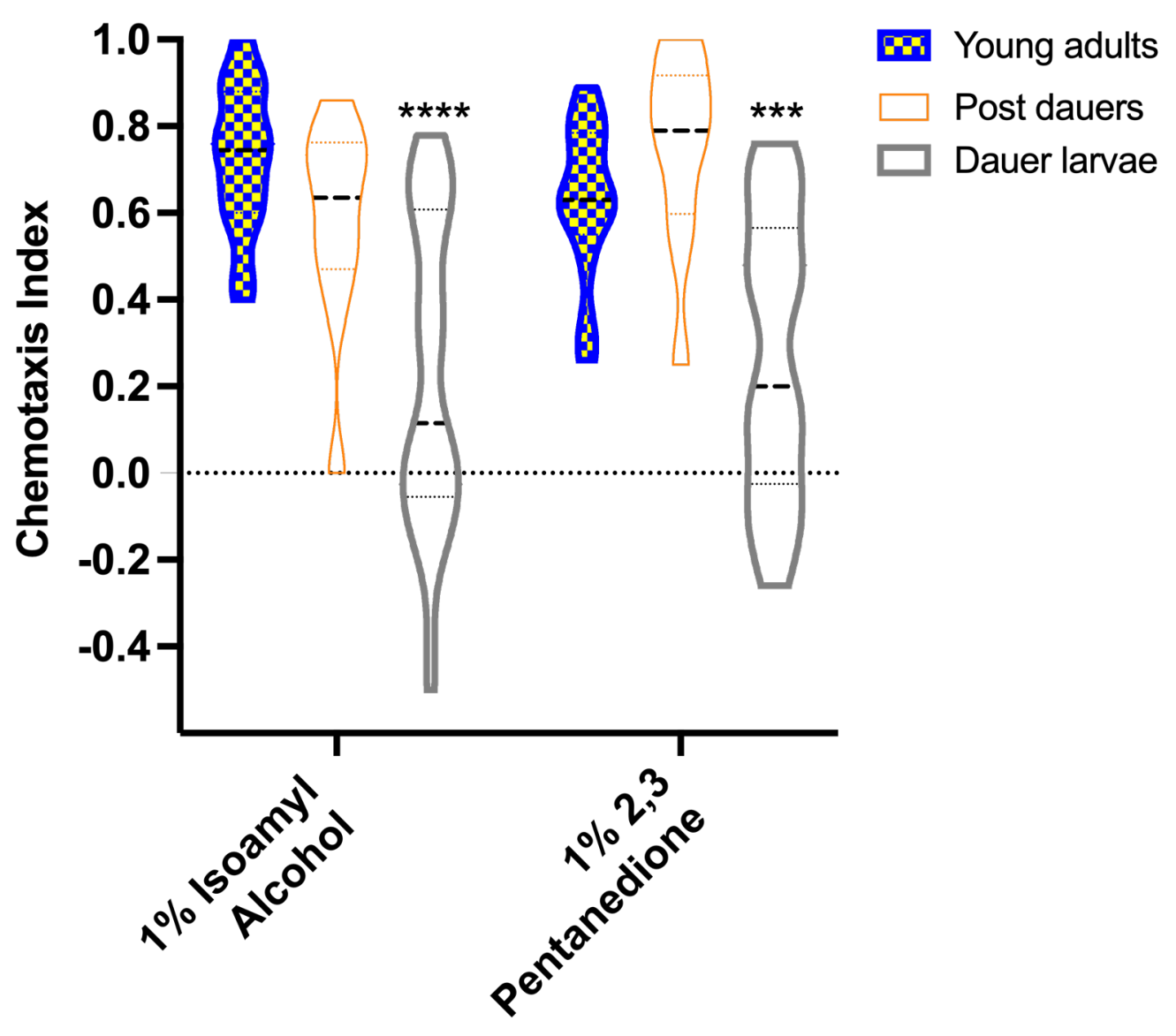

SI Figure 4. Olfactory response of $C$. elegans post-dauer adults. The chemotaxis response of wild-type post-dauer young adults in C. elegans resemble never-dauer young adults but not dauer larvae. IAA: One-way ANOVA against adults with Kruskal-Wallis test. Pentanedione: One-way ANOVA against adults with Sidak's post-hoc test. ${ }^{* * *} P<0.001 ;{ }^{* * * *} P<0.0001$. 


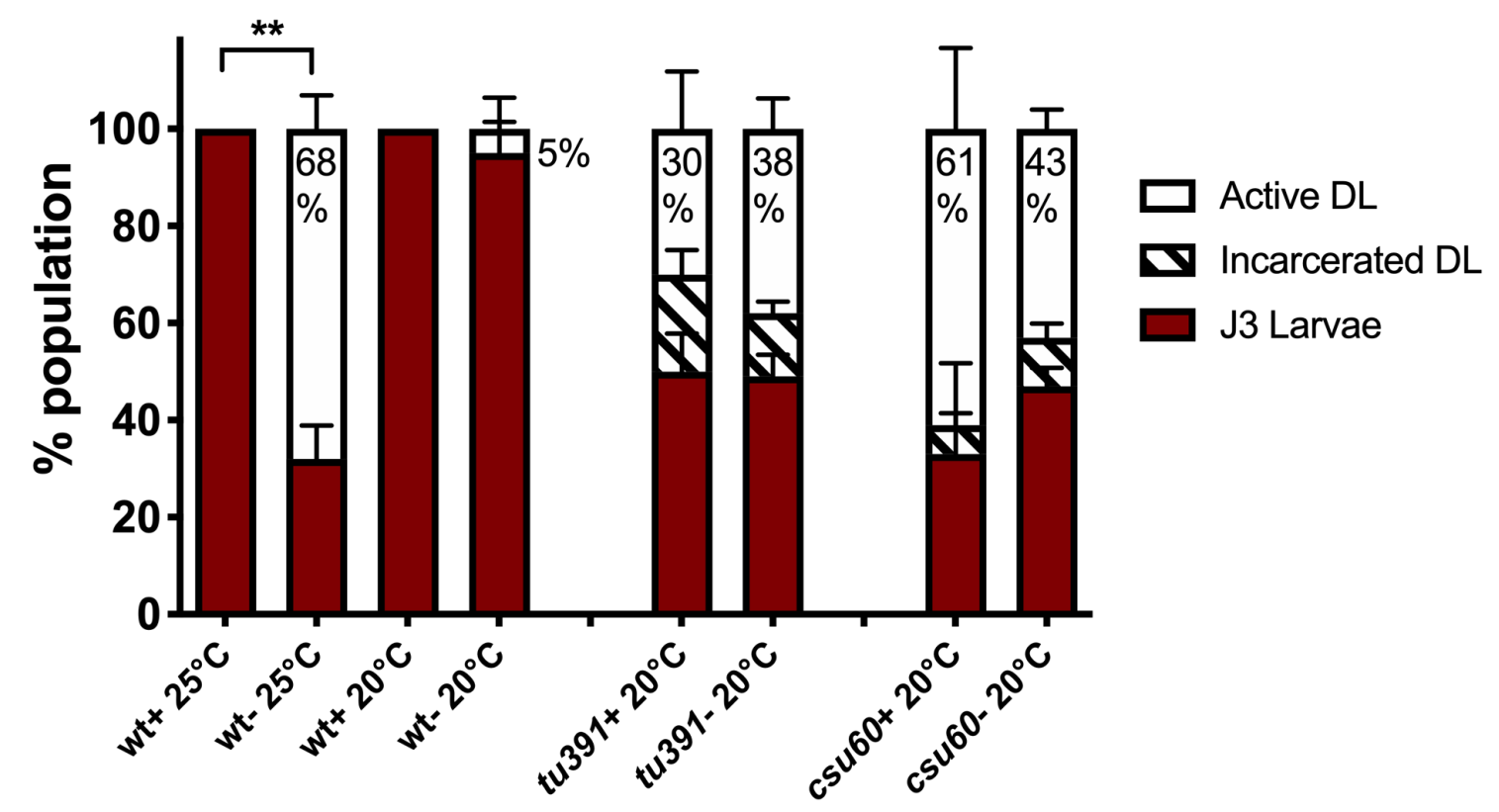

SI Figure 5. Effects of cholesterol removal in Daf-c mutants. The progeny of worms that were maintained for two generations on NGM plates with $(+)$ or without $(-)$ the normal cholesterol supplement in the medium were scored for the formation of active $\mathrm{DL}$. At $25^{\circ} \mathrm{C}$, the removal of cholesterol greatly increased dauer formation in wildtype worms but not in Daf-c mutants at $20^{\circ} \mathrm{C}$ (Comparisons were done at $20^{\circ} \mathrm{C}$, since $>92 \%$ of $t u 391$ and csu 60 form DL already at $25^{\circ} \mathrm{C}$ ). The percentage of active $\mathrm{DL}$ is indicated. For each condition, $4-5$ assays were performed over two trials. Difference in active DL in wildtype: One-way ANOVA with Kruskal-Wallis test. ${ }^{*} P<0.01$. Difference in active $\mathrm{DL}$ in each Daf-c mutant: t-test with MannWhitney test detected no significant difference. 

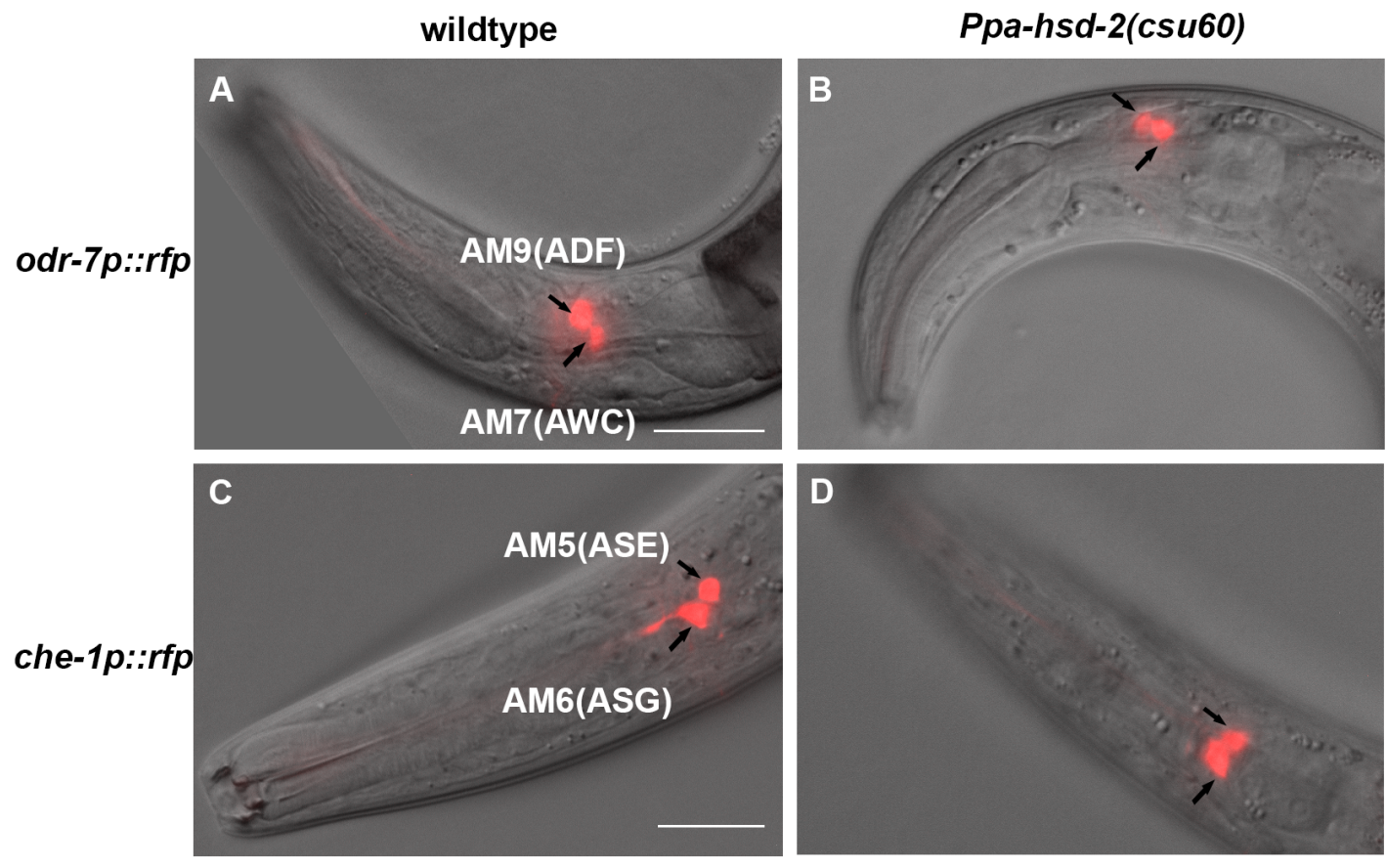

\begin{abstract}
SI Figure 6. Amphid neuron marker expressions for two reporters are wildtype in Ppa-hsd-2(csu60). Representative overlay images of adult hermaphrodites expressing the Ppa-odr-7p::Ifp reporter in AM9(ADF) and AM7(AWC) neurons of (A) wild-type PS312 and (B) $h s d-2$ (csu60) mutant. Overlay images of adult hermaphrodites expressing the Ppa-che-1p::Ifp reporter in AM5(ASE) and AM6(ASG) neurons of (C) wild-type PS312 and (D) hsd-2(csu60) mutant. Anterior is left and dorsal is up. Scale bars represent each reporter strain: $20 \mu \mathrm{m}$.
\end{abstract}




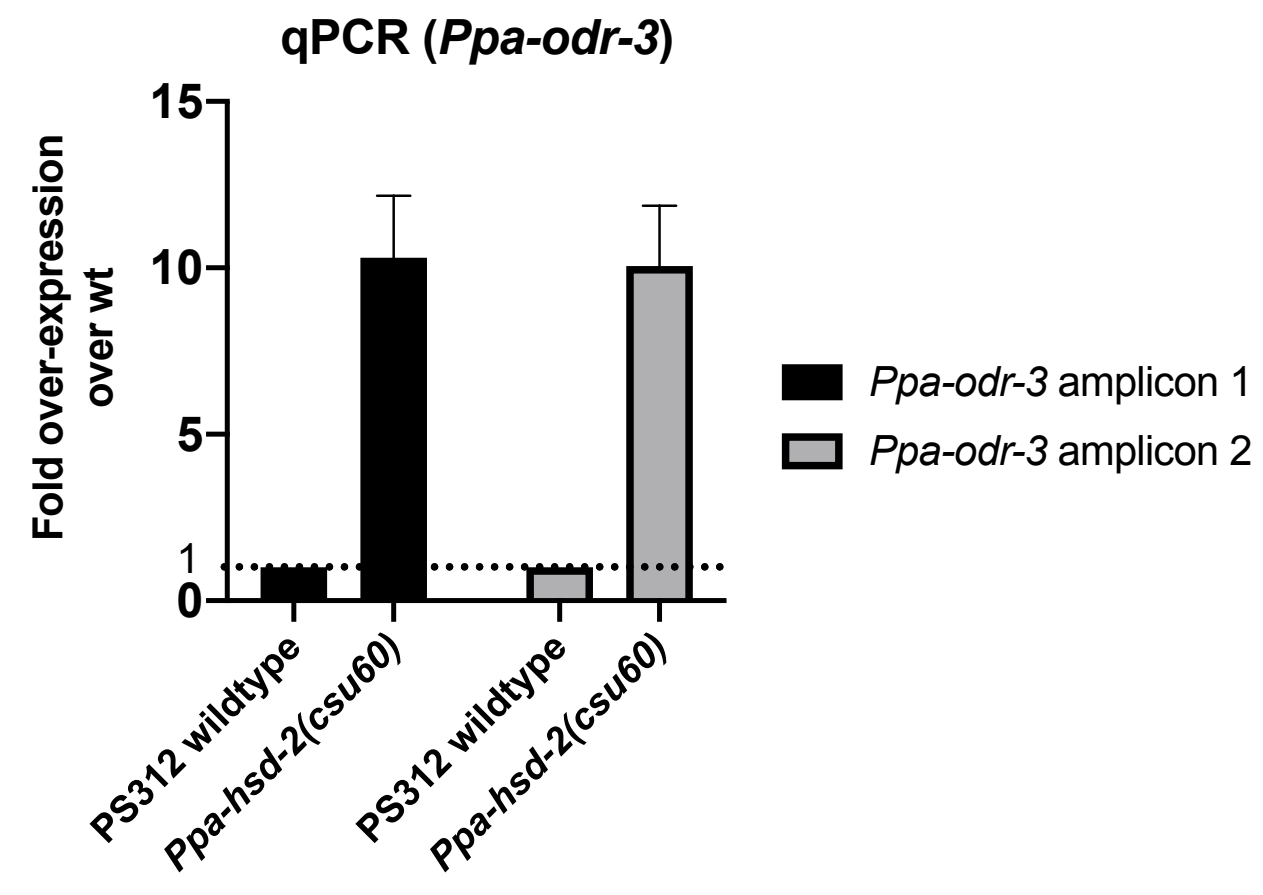

SI Figure 7. RT-qPCR results of Ppa-odr-3 expression. Over-expression of Ppa-odr-3 in $h s d$-2(csu60) is $\sim 10$-fold more abundant than wild-type PS312 (arbitrarily set as one). Data represents 12 technical replicates from two biological RNA extraction replicates. Error bars denote s.e.m. 

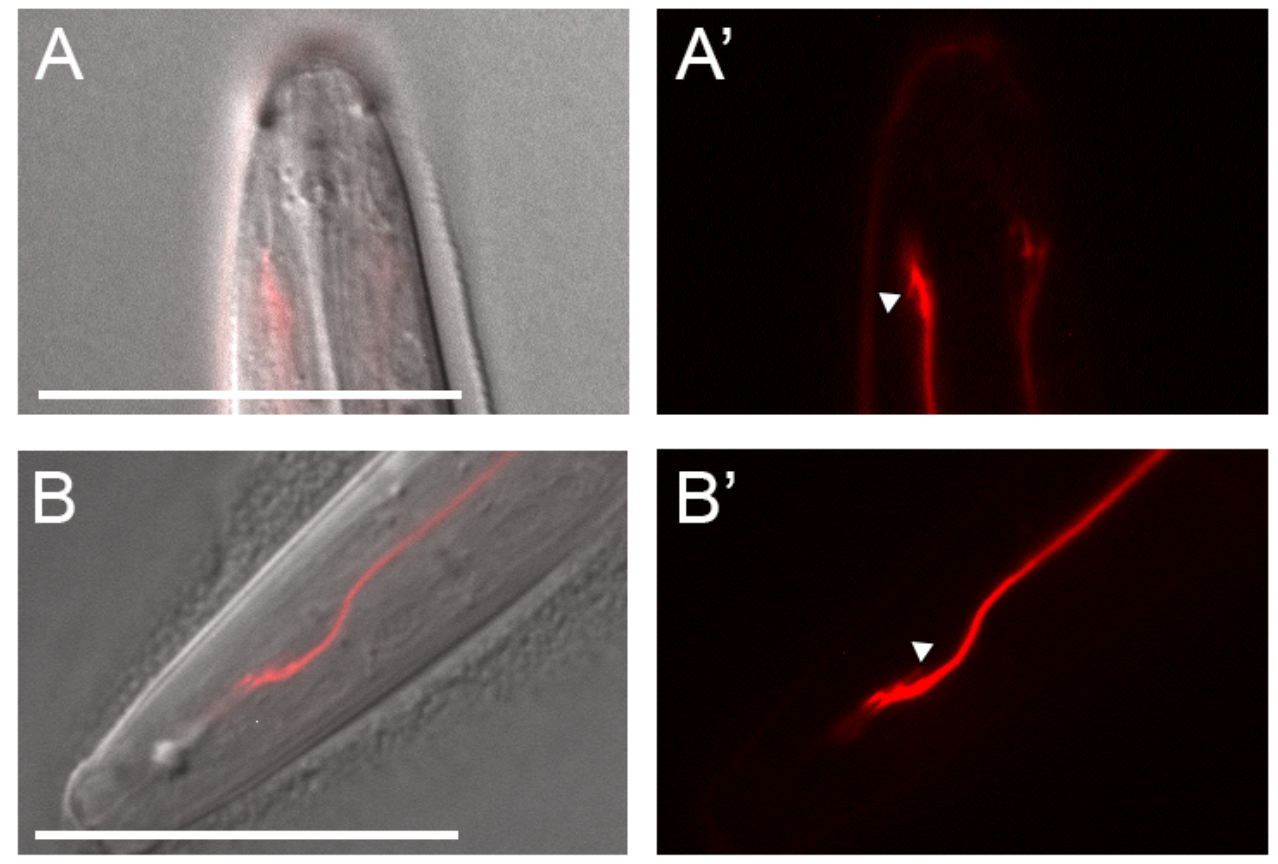

SI Figure 8. Posterior protrusions in Ppa-odr-3p::rfp dendritic endings of Ppa-hsd-2(csu60) dauer larvae. (A-B) Overlay images of additional examples of unusual dendritic ends found in csu60 dauer larvae. (A' and B') RFP fluorescence. Triangle points to a type of posterior protrusion observed in 3 out of 17 csu60 DL but not in wild-type DL. Anterior is up for (A), which is also a ventral view, and left for (B). Scale bar: $25 \mu \mathrm{m}$. 
bioRxiv preprint doi: https://doi.org/10.1101/2020.03.30.016311; this version posted March 30,2020. The copyright holder for this preprint (which was not certified by peer review) is the author/funder, who has granted bioRxiv a license to display the preprint in perpetuity. It is made available under aCC-BY 4.0 International license. 


\section{References}

[1] Baugh LR, DeModena J, Sternberg PW. RNA Pol II Accumulates at Promoters of Growth Genes During Developmental Arrest. Science 2009;324:92-4.

[2] Zaslaver A, Baugh LR, Sternberg PW. Metazoan Operons Accelerate Recovery from Growth-Arrested States. Cell 2011;145:981-92. doi:10.1016/j.cell.2011.05.013.

[3] Albert PS, Riddle DL. Developmental Alterations in Sensory Neuroanatomy of the Caenorhabditis elegans Dauer Larva. Journal of Comparative Neurology 1983;219:461-81. doi:10.1002/cne.902190407.

[4] Procko C, Lu Y, Shaham S. Glia delimit shape changes of sensory neuron receptive endings in C. elegans. Development 2011;138:137181. doi:10.1242/dev.058305.

[5] Li J, Zhu XD, Ashton FT, Gamble HR, Schad GA. Sensory neuroanatomy of a passively ingested nematode parasite, Haemonchus contortus: Amphidial neurons of the third-stage larva. Journal of Parasitology 2001;87:65-72.

[6] Lee JS, Shih P-Y, Schaedel ON, Quintero-Cadena P, Rogers AK, Sternberg PW. FMRFamide-like peptides expand the behavioral repertoire of a densely connected nervous system. Proceedings of the National Academy of Sciences 2017;114:E10726-35. doi:10.1073/pnas.1710374114.

[7] Vidal B, Aghayeva U, Sun H, Wang C, Glenwinkel L, Bayer EA, et al. An atlas of Caenorhabditis elegans chemoreceptor expression. PLoS Biol 2018;16:e2004218. doi:10.1371/journal.pbio.2004218.s004.

[8] Ogawa A, Streit A, Antebi A, Sommer RJ. A conserved endocrine mechanism controls the formation of dauer and infective larvae in nematodes. Curr Biol 2009;19:67-71. doi:10.1016/j.cub.2008.11.063.

[9] Ogawa A, Bento G, Bartelmes G, Dieterich C, Sommer RJ. Pristionchus pacificus daf-16 is essential for dauer formation but dispensable for mouth form dimorphism. Development 2011;138:1281-4. doi:10.1242/dev.058909.

[10] Ma G, Wang T, Korhonen PK, Young ND, Nie S, Ang C-S, et al. Dafachronic acid promotes larval development in Haemonchus contortus by modulating dauer signalling and lipid metabolism. PLoS Pathog 2019;15:e1007960. doi:10.1371/journal.ppat.1007960.s007.

[11] Fielenbach N, Antebi A. C. elegans dauer formation and the molecular basis of plasticity. Genes Dev 2008;22:2149-65.

doi:10.1101/gad.1701508.

[12] Ren P, Lim CS, Johnsen R, Albert PS, Pilgrim D, Riddle DL. Control of C. elegans larval development by neuronal expression of a TGF-beta homolog. Science 1996;274:1389-91.

[13] Gems D, Sutton AJ, Sundermeyer ML, Albert PS, King KV, Edgley ML, et al. Two Pleiotropic Classes of daf-2 Mutation Affect Larval Arrest, Adult 
Behavior, Reproduction and Longevity in Caenorhabditis elegans. Genetics 1998;150:129.

[14] Ogg S, Paradis S, Gottlieb S, Patterson GI, Lee L, Tissenbaum HA, et al. The Fork head transcription factor DAF-16 transduces insulin-like metabolic and longevity signals in C. elegans. Nature 1997;389:994-9.

[15] Dumas KJ, Guo C, Wang X, Burkhart KB, Adams EJ, Alam H, et al. Developmental Biology. Developmental Biology 2010;340:605-12. doi:10.1016/j.ydbio.2010.02.022.

[16] Schaedel ON, Gerisch B, Antebi A, Sternberg PW. Hormonal Signal Amplification Mediates Environmental Conditions during Development and Controls an Irreversible Commitment to Adulthood. PLoS Biol 2012;10:e1001306. doi:10.1371/journal.pbio.1001306.s009.

[17] Werner MS, Sieriebriennikov B, Loschko T, Namdeo S, Lenuzzi M, Dardiry M, et al. Environmental influence on. Sci Rep 2017:1-12. doi:10.1038/s41598-017-07455-7.

[18] Peckol EL, Zallen JA, Yarrow JC, Bargmann Cl. Sensory activity affects sensory axon development in C. elegans. Development 1999;126:1891902.

[19] Bhattacharya A, Aghayeva U, Berghoff EG, Hobert O. Plasticity of the Electrical Connectome of C. elegans. Cell 2019;176:1174-1189.e16. doi:10.1016/j.cell.2018.12.024.

[20] Hallem EA, Sternberg PW. Acute carbon dioxide avoidance in Caenorhabditis elegans. Proc Natl Acad Sci USa 2008;105:8038-43. doi:10.1073/pnas.0707469105.

[21] Hallem EA, Spencer WC, McWhirter RD, Zeller G, Henz SR, R H a tsch $\mathrm{G}$, et al. Receptor-type guanylate cyclase is required for carbon dioxide sensation by Caenorhabditis elegans. Proc Natl Acad Sci USa 2011;108:254-9. doi:10.1073/pnas.1017354108.

[22] Castelletto ML, Gang SS, Okubo RP, Tselikova AA, Nolan TJ, Platzer EG, et al. Diverse Host-Seeking Behaviors of Skin-Penetrating Nematodes. PLoS Pathog 2014;10:e1004305. doi:10.1371/journal.ppat.1004305.s016.

[23] Bargmann Cl. Genetic and cellular analysis of behavior in C. elegans. Annu Rev Neurosci 1993;16:47-71. doi:10.1146/annurev.ne.16.030193.000403.

[24] Bargmann Cl, Hartwieg E, Horvitz HR. Odorant-selective genes and neurons mediate olfaction in C. elegans. Cell 1993;74:515-27.

[25] Jovelin R, Ajie BC, Phillips, P. C. Molecular evolution and quantitative variation for chemosensory behaviour in the nematode genus Caenorhabditis. Molecular Ecology 2003;12:1325-37.

[26] O'Halloran DM, Burnell AM. An investigation of chemotaxis in the insect parasitic nematode Heterorhabditis bacteriophora. Parasitology 2003;127:375-85.

[27] Hallem EA, Dillman AR, Hong AV, Zhang Y, Yano JM, DeMarco SF, et al. A sensory code for host seeking in parasitic nematodes. Curr Biol 2011;21:377-83. doi:10.1016/j.cub.2011.01.048. 
[28] Okumura E, Yoshiga T. Host orientation using volatiles in the phoretic nematode Caenorhabditis japonica. Journal of Experimental Biology 2014;217:3197-9. doi:10.1242/jeb.105353.

[29] Baiocchi T, Lee G, Choe D-H, Dillman AR. Host seeking parasitic nematodes. Sci Rep 2017:1-13. doi:10.1038/s41598-017-06620-2.

[30] Turlings TCJ, Hiltpold I, Rasmann S. The importance of root-produced volatiles as foraging cues for entomopathogenic nematodes. Plant and Soil 2012;358:51-60.

[31] Bryant AS, Hallem EA. IJP: Drugs and Drug Resistance. IJP: Drugs and Drug Resistance 2018;8:496-510. doi:10.1016/j.ijpddr.2018.10.008.

[32] Weller AM, Mayer WE, Rae R, Sommer RJ. Quantitative assessment of the nematode fauna present on Geotrupes dung beetles reveals speciesrich communities with a heterogeneous distribution. J Parasitol 2010;96:525-31. doi:10.1645/GE-2319.1.

[33] Sommer RJ, Mayer MG. Toward a Synthesis of Developmental Biology with Evolutionary Theory and Ecology. Annu Rev Cell Dev Biol 2015;31:453-71. doi:10.1146/annurev-cellbio-102314-112451.

[34] Meyer JM, Baskaran P, Quast C, Susoy V, rodelsperger C, Glöckner FO, et al. Succession and dynamics of Pristionchusnematodes and their microbiome during decomposition of Oryctes borbonicuson La Réunion Island. Environ Microbiol 2017;19:1476-89. doi:10.1016/j.jip.2008.02.011.

[35] Penkov S, Ogawa A, Schmidt U, Tate D, Zagoriy V, Boland S, et al. a wax ester promotes collective host finding in the nematode Pristionchus pacificus. Nature Chemical Biology 2014:1-7.

doi:10.1038/nchembio.1460.

[36] Mayer MG, Sommer RJ. Natural variation in Pristionchus pacificus dauer formation reveals cross-preference rather than self-preference of nematode dauer pheromones. Proc Biol Sci 2011;278:2784-90. doi:10.1098/rspb.2010.2760.

[37] Bento G, Ogawa A, Sommer RJ. Co-option of the hormone-signalling module dafachronic acid-DAF-12 in nematode evolution. Nature 2010;466:494-7. doi:10.1038/nature09164.

[38] Kroetz SM, Srinivasan J, Yaghoobian J, Sternberg PW, Hong RL. The cGMP Signaling Pathway Affects Feeding Behavior in the Necromenic Nematode Pristionchus pacificus. PLoS One 2012;7:e34464. doi:10.1371/journal.pone.0034464.

[39] Hong RL, Sommer RJ. Chemoattraction in Pristionchus nematodes and implications for insect recognition. Curr Biol 2006;16:2359-65. doi:10.1016/j.cub.2006.10.031.

[40] Golden JW, Riddle DL. A pheromone influences larval development in the nematode Caenorhabditis elegans. Science 1982;218:578.

[41] Jeong PY, Jung M, Yim YH, Kim H, Park M, Hong EM, et al. Chemical structure and biological activity of the Caenorhabditis elegans dauerinducing pheromone. Nature 2005;433:541-5.

[42] Butcher RA, Fujita M, Schroeder FC, Clardy J. Small-molecule pheromones that control dauer development in Caenorhabditis elegans. 
Nature Chemical Biology 2007;3:420-2.

[43] Schroeder FC. Modular Assembly of Primary Metabolic Building Blocks: A Chemical Language in C. elegans. Chemistry \& Biology 2015;22:7-16. doi:10.1016/j.chembiol.2014.10.012.

[44] Golden JW, Riddle DL. A gene affecting production of the Caenorhabditis elegans dauer-inducing pheromone. Molecular and General Genetics MGG 1985;198:534-6.

[45] Markov GV, Meyer JM, Panda O, Artyukhin AB, Claaßen M, Witte H, et al. Functional conservation and divergence of daf-22 paralogs in $\mathrm{P}$. pacificus dauer development. Mol Biol Evol 2016.

doi:10.1093/molbev/msw090.

[46] Sieriebriennikov B, Markov GV, Witte H, Sommer RJ. The Role of DAF21/Hsp90 in Mouth-Form Plasticity in Pristionchus pacificus. Mol Biol Evol 2017;34:1644-53. doi:10.1093/molbev/msx106.

[47] Moreno E, Lenuzzi M, rodelsperger C, Prabh N, Witte H, Roeseler W, et al. DAF-19/RFX controls ciliogenesis and influences oxygen-induced social behaviors in Pristionchus pacificus. Evol Dev 2018;20:233-43. doi:10.1016/j.neuron.2009.02.013.

[48] Mayer MG, rodelsperger C, Witte H, Riebesell M, Sommer RJ. The Orphan Gene dauerless Regulates Dauer Development and Intraspecific Competition in Nematodes by Copy Number Variation. PLoS Genet 2015;11:e1005146. doi:10.1371/journal.pgen.1005146.s003.

[49] Yochem J, Tuck S, Greenwald I, Han M. A gp330/megalin-related protein is required in the major epidermis of Caenorhabditis elegans for completion of molting. Development 1999;126:597-606.

[50] Matyash V, Entchev EV, Mende F, Wilsch-Bräuninger M, Thiele C, Schmidt AW, et al. Sterol-derived hormone(s) controls entry into diapause in Caenorhabditis elegans by consecutive activation of DAF-12 and DAF16. PLoS Biol 2004;2:e280. doi:10.1371/journal.pbio.0020280.

[51] Motola DL, Cummins CL, Rottiers V, Sharma KK, Li TT, Li Y, et al. Identification of ligands for DAF-12 that govern Dauer formation and reproduction in C. elegans. Cell 2006;124:1209-23.

[52] Kiontke K, Sudhaus W. Ecology of Caenorhabditis species. WormBook, Ed the C Elegans Research Community, Wormbook 2006.

[53] Campbell LR, Gaugler R. Mechanisms for exsheathment of entomopathogenic nematodes. International Journal for Parasitology 1991;21:219-24.

[54] Menti H, Wright DJ, Perry RN. Dessication survival of populations of the entomopathogenic nematodes Steinernema feltiae and Heterohabditis megidis from Greece and the uK. J Helminthol 1997;71:41-6.

[55] Swanson MM, Riddle DL. Critical periods in the development of the Caenorhabditis elegans dauer larva. Developmental Biology 1981;84:2740.

[56] Golden JW, Riddle DL. The Caenorhabditis elegans dauer larva: developmental effects of pheromone, food, and temperature. Developmental Biology 1984;102:368-78. 
[57] Ailion $\mathrm{M}$, Thomas $\mathrm{JH}$. Dauer formation induced by high temperatures in Caenorhabditis elegans. Genetics 2000;156:1047-67.

[58] Rivard L, Srinivasan J, Stone A, Ochoa S, Sternberg PW, Loer CM. A comparison of experience-dependent locomotory behaviors and biogenic amine neurons in nematode relatives of Caenorhabditis elegans. BMC Neurosci 2010;11:22. doi:10.1186/1471-2202-11-22.

[59] Han Z, Boas S, Schroeder NE. Unexpected Variation in Neuroanatomy among Diverse Nematode Species. Front Neuroanat 2016;9:296. doi:10.1002/cne.22637.

[60] Hong RL, Riebesell M, Bumbarger DJ, Cook SJ, Carstensen HR, Sarpolaki T, et al. Evolution of neuronal anatomy and circuitry in two highly divergent nematode species. eLife 2019;8.

doi:10.7554/eLife.47155.

[61] Starich TA, Herman RK, Kari CK, Yeh WH, Schackwitz WS, Schuyler $\mathrm{MW}$, et al. Mutations affecting the chemosensory neurons of Caenorhabditis elegans. Genetics 1995;139:171-88.

[62] Perkins LA, Hedgecock EM, Thomson JN, Culotti JG. Mutant sensory cilia in the nematode Caenorhabditis elegans. Dev Biol 1986;117:456-87.

[63] Malone EA, Thomas JH. A screen for nonconditional dauer-constitutive mutations in Caenorhabditis elegans. Genetics 1994;136:879-86.

[64] Swoboda P, Adler HT, Thomas JH. The RFX-type transcription factor DAF-19 regulates sensory neuron cilium formation in $\mathrm{C}$. elegans. Molecular Cell 2000;5:411-21.

[65] Nuttley WM, Harbinder S, van der Kooy D. Regulation of distinct attractive and aversive mechanisms mediating benzaldehyde chemotaxis in Caenorhabditis elegans. Learn Mem 2001;8:170-81. doi:10.1101/lm.36501.

[66] Hong RL, Witte H, Sommer RJ. Natural variation in Pristionchus pacificus insect pheromone attraction involves the protein kinase EGL-4. Proc Natl Acad Sci USa 2008;105:7779-84. doi:10.1073/pnas.0708406105.

[67] Herrmann M, Mayer W, Hong RL, Kienle S, Minasaki R, sommer RJ. The nematode Pristionchus pacificus (Nematoda: Diplogastridae) is associated with the oriental beetle Exomala orientalis (Coleoptera: Scarabaeidae) in Japan. Zool Sci 2007;24:883-9. doi:10.2108/zsj.24.883.

[68] Cinkornpumin JK, Wisidagama DR, Rapoport V, Go JL, Dieterich C, Wang $X$, et al. A host beetle pheromone regulates development and behavior in the nematode Pristionchus pacificus. eLife 2014;3. doi:10.7554/eLife.03229.

[69] Sims JR, Ow MC, Nishiguchi MA, Kim K, Sengupta P, Hall SE. Developmental programming modulates olfactory behavior in C. elegans via endogenous RNAi pathways 2016:1-26. doi:10.7554/eLife.11642.

[70] Pradhan S, Quilez S, Homer K, Hendricks M. Environmental Programming of Adult Foraging Behavior in C. elegans. Current Biology 2019;29:2867-2879.e4. doi:10.1016/j.cub.2019.07.045.

[71] Felix MA, Braendle C. The natural history of Caenorhbditis elegans. Current Biology 2010;20:R965-9. 
[72] Kimura KD, Tissenbaum HA, Liu Y, Ruvkun G. daf-2, an Insulin Receptor-Like Gene That Regulates Longevity and Diapause in Caenorhabditis elegans. Science 1997;277:942.

[73] Patel DS, Fang LL, Svy DK, Ruvkun G, Li W. Genetic identification of HSD-1, a conserved steroidogenic enzyme that directs larval development in Caenorhabditis elegans. Development 2008;135:223949. doi:10.1242/dev.016972.

[74] Baskaran P, roedelsperger C, Prabh N, Serobyan V, Markov GV, Hirsekorn A, et al. Ancient gene duplications have shaped developmental stage-specific expressionin Pristionchus pacificus. BMC Evol Biol 2015:1-12. doi:10.1186/s12862-015-0466-2.

[75] Brenner S. The genetics of Caenorhabditis elegans. Genetics 1974;77:71-94.

[76] Sommer RJ. Pristionchus pacificus. WormBook 2006:1-8. doi:10.1895/wormbook.1.102.1.

[77] Fürst von Lieven A. The embryonic moult in diplogastrids (Nematoda) homology of developmental stages and heterochrony as a prerequisite for morphological diversity. Zoologischer Anzeiger - a Journal of Comparative Zoology 2005;244:79-91. doi:10.1016/j.jcz.2005.05.001.

[78] Lewis VM, Hong RL. Conserved behavioral and genetic mechanisms in the pre-hatching molt of the nematode Pristionchus pacificus 2014;5:1-8. doi:10.1186/2041-9139-5-31.

[79] Cullen PJ, CRanston PR. The Insects: An Outline of Entomology. third. Blackwell Publishing; 2009.

[80] Patel MN, Wright DJ. The ultrastructure of the cuticle and sheath of infective juveniles of entomopathogenic steinernematid nematodes. $J$ Helminthol 1998;72:257-66.

[81] Wharton DA. Ultrastructural changes associated with exsheathment of infective juveniles of Haemonchus contortus. Parasitology 2020;103:41320. doi:10.1017/S003118200005993X.

[82] Campbell LR, Gaugler R. Effect of Exsheathment on Motility and Pathogenicity of Two Entomopathogenic Nematode Species. Journal of Nematology 1992;24:365-70.

[83] Riddle DL, Swanson MM, Albert PS. Interacting genes in nematode dauer larva formation. Nature 1981;290:668-71.

[84] Kelley M, Yochem J, Krieg M, Calixto A, Heiman MG, Kuzmanov A, et al. FBN-1, a fibrillin-related protein, is required for resistance of the epidermis to mechanical deformation during $\mathrm{C}$. elegans embryogenesis. eLife 2015;4. doi:10.7554/eLife.06565.

[85] Sagasti A, Hobert O, Troemel ER, Ruvkun G, Bargmann Cl. Alternative olfactory neuron fates are specified by the LIM homeobox gene lim- 4 . Genes Dev 1999;13:1794-806.

[86] Sinha A, Sommer RJ, Dieterich C. Divergent gene expression in the conserved dauer stage of the nematodes Pristionchus pacificus and Caenorhabditis elegans. BMC Genomics 2012;13:1-17. doi:10.1186/1471-2164-13-254. 
[87] Ragsdale EJ, Müller MR, roedelsperger C, Sommer RJ. A developmental switch coupled to the evolution of plasticity acts through a sulfatase. Cell 2013;155:922-33. doi:10.1016/j.cell.2013.09.054.

[88] Perens EA, Shaham S. C-elegans daf-6 encodes a patched-related protein required for lumen formation. Dev Cell 2005;8:893-906. doi:10.1016/j.devcel.2005.03.009.

[89] Rottiers V, Motola DL, Gerisch B, Cummins CL, Nishiwaki K, Mangelsdorf DJ, et al. Hormonal control of C-elegans dauer formation and life span by a Rieske-like oxygenase. Developmental Cell 2006;10:473-82.

[90] Parihar M, Minton RL, Flowers S, Holloway A, Morehead BE, Paille J, et al. The genome of the nematode Pristionchus pacificus encodes putative homologs of RXR/Usp and EcR. Gen Comp Endocrinol 2010;167:11-7. doi:10.1016/j.ygcen.2010.02.005.

[91] Marie-Anne Félix MAJ-CHARJW. Pristionchus nematodes occur frequently in diverse rotting vegetal substrates and are not exclusively necromenic, while Panagrellus redivivoides is found specifically in rotting fruits. PLoS One 2018:1-21. doi:10.1371/journal.pone.0200851.

[92] Lee J, Jee C, Mclntire SL. Ethanol preference in C. elegans. Genes, Brain and Behavior 2009;8:578-85. doi:10.1038/nature04216.

[93] Margie O, Palmer C, Chin-Sang I. C. elegans Chemotaxis Assay. J Vis Exp 2013. doi:10.3791/50069.

[94] Carrillo MA, Guillermin ML, Rengarajan S, Okubo RP, Hallem EA. O2Sensing Neurons Control CO2 Response in C. elegans. Journal of Neuroscience 2013;33:9675-83. doi:10.1523/JNEUROSCI.454112.2013.

[95] Doitsidou M, Poole RJ, Sarin S, Bigelow H, Hobert O. C. elegans Mutant Identification with a One-Step Whole-Genome-Sequencing and SNP Mapping Strategy. PLoS One 2010;5:e15435. doi:10.1371/journal.pone.0015435.s002.

[96] Schuster LN, Sommer RJ. Expressional and functional variation of horizontally acquired cellulases in the nematode Pristionchus pacificus. Gene 2012;506:274-82. doi:10.1016/j.gene.2012.07.013.

[97] Pfaffl MW. A new mathematical model for relative quantification in realtime RT-PCR. Nucleic Acids Res 2001;29:e45-5.

[98] Dereeper A, Guignon V, Blanc G, Audic S, Buffet S, Chevenet F, et al. Phylogeny.fr: robust phylogenetic analysis for the non-specialist. Nucleic Acids Res 2008;36:W465-9. doi:10.1093/bioinformatics/bti263. 